\title{
Topological protection, disorder, and interactions: Survival at the surface of three-dimensional topological superconductors
}

\author{
Matthew S. Foster," Hong-Yi Xie, and Yang-Zhi Chou \\ Department of Physics and Astronomy, Rice University, Houston, Texas 77005, USA \\ (Received 31 March 2014; revised manuscript received 13 April 2014; published 30 April 2014)
}

\begin{abstract}
We consider the interplay of disorder and interactions upon the gapless surface states of 3D topological superconductors. The combination of topology and superconducting order inverts the action of time-reversal symmetry, so that extrinsic time-reversal invariant surface perturbations appear only as "pseudomagnetic" fields (Abelian and non-Abelian vector potentials, which couple to spin and valley currents). The main effect of disorder is to induce multifractal scaling in surface state wave functions. These critically delocalized, yet strongly inhomogeneous states renormalize interaction matrix elements relative to the clean system. We compute the enhancement or suppression of interaction scaling dimensions due to the disorder exactly, using conformal field theory. We determine the conditions under which interactions remain irrelevant in the presence of disorder for symmetry classes AIII and DIII. In the limit of large topological winding numbers (many surface valleys), we show that the effective field theory takes the form of a Finkel'stein nonlinear sigma model, augmented by the Wess-Zumino-Novikov-Witten term. The sigma model incorporates interaction effects to all orders and provides a framework for a controlled perturbative expansion; the inverse spin or thermal conductance is the small parameter. For class DIII, we show that interactions are always irrelevant, while in class AIII, there is a finite window of stability, controlled by the disorder. Outside of this window, we identify new interaction-stabilized fixed points.
\end{abstract}

DOI: 10.1103/PhysRevB.89.155140

PACS number(s): 64.60.al, 05.30.Rt, 73.20.Fz

\section{INTRODUCTION}

The discoveries of graphene, 2D and 3D topological insulators have cemented "Dirac materials" as a new physics frontier. Much recent interest has pivoted to employing these as platforms for topological superconductivity and Majorana fermion zero modes, usually through proximity coupling to an ordinary $s$-wave superconductor [1]. On the other hand, the discovery of bulk 3D topological superconductors [2-15] would give access to a fundamentally different type of Dirac material, with features not easily attainable elsewhere.

Bulk topological superconductors (TSCs) should solve the energy mismatch problem that often plagues other Dirac materials. In a topological insulator, the chemical potential may reside in the gap, in the valence or conduction bands, and the same is true of the surface state Dirac point. In general, there is no fundamental mechanism to pin the chemical potential in the gap and/or align it to the Dirac point [6]. TSCs are predicted to host Majorana bands of topologically protected quasiparticles at the material surface $[2,6,16]$. These surface bands penetrate the bulk superconducting gap, and the chemical potential appears precisely at the Dirac point of the surface band (inside the gap), due to particle-hole symmetry.

Bulk TSCs can be realized in three of the five topological classes of the Altland-Zirnbauer scheme in three dimensions; these are denoted DIII, AIII, and CI [2,3,5]. In order for these to be topological, time-reversal symmetry must be preserved in the bulk. The three classes differ by the degree of spin rotational symmetry. Class DIII has no spin symmetry, as occurs if strong spin-orbit coupling is present [9,11-14]. Class AIII has spin U(1) symmetry, as might arise in a time-reversal

*matthew.foster@ rice.edu invariant spin-triplet $p$-wave superconductor. Class CI has full spin $\mathrm{SU}(2)$ symmetry and spin singlet pairing $[4,10,15]$.

A remarkable feature of topological superconductors is that the effects of time-reversal symmetry are "inverted": external perturbations of the surface that respect time-reversal invariance appear as pseudomagnetic fields (Abelian or nonAbelian vector potentials) [2]. Because defects and disorder are inevitable at material interfaces, a generic TSC surface will realize a kind of quenched (2+1)-D quantum chromodynamics, wherein massless Dirac quasiparticles navigate a landscape of frozen gauge fluctuations $[2,15,17]$. (There is one exception, which is a single Dirac valley in class DIII [16]. In this case there are no conserved currents except energy, and disorder can enter only through a modulation of the Fermi velocity [18].) The physics underlying this unusual version of time-reversal symmetry is the unique conflagration of superconducting order and topology: surface vector potentials couple to time-reversal even spin and valley currents. The corresponding densities are odd. Topological protection forbids the appearance of a Dirac mass.

Equally remarkable is the response of the surface quasiparticles to nonmagnetic disorder. Topological protection implies that at least one surface wave function must remain delocalized. As first demonstrated 20 years ago $[19,20]$, the problem of 2D Dirac fermions coupled to quenched random (Abelian or non-Abelian) vector potentials is solvable via conformal field theory. Wave functions near zero energy are critically delocalized, and their universal statistical properties such as the multifractal [21] spectrum of local density of states fluctuations $[22,23]$ can be computed exactly $[15,19,20,24$ 36].

In this paper, we compute universal properties of and construct global phase diagrams for topological superconductor surface states. We consider the simultaneous effects of both disorder and interactions. We determine properties of the critically delocalized surface states in the absence of interactions, 

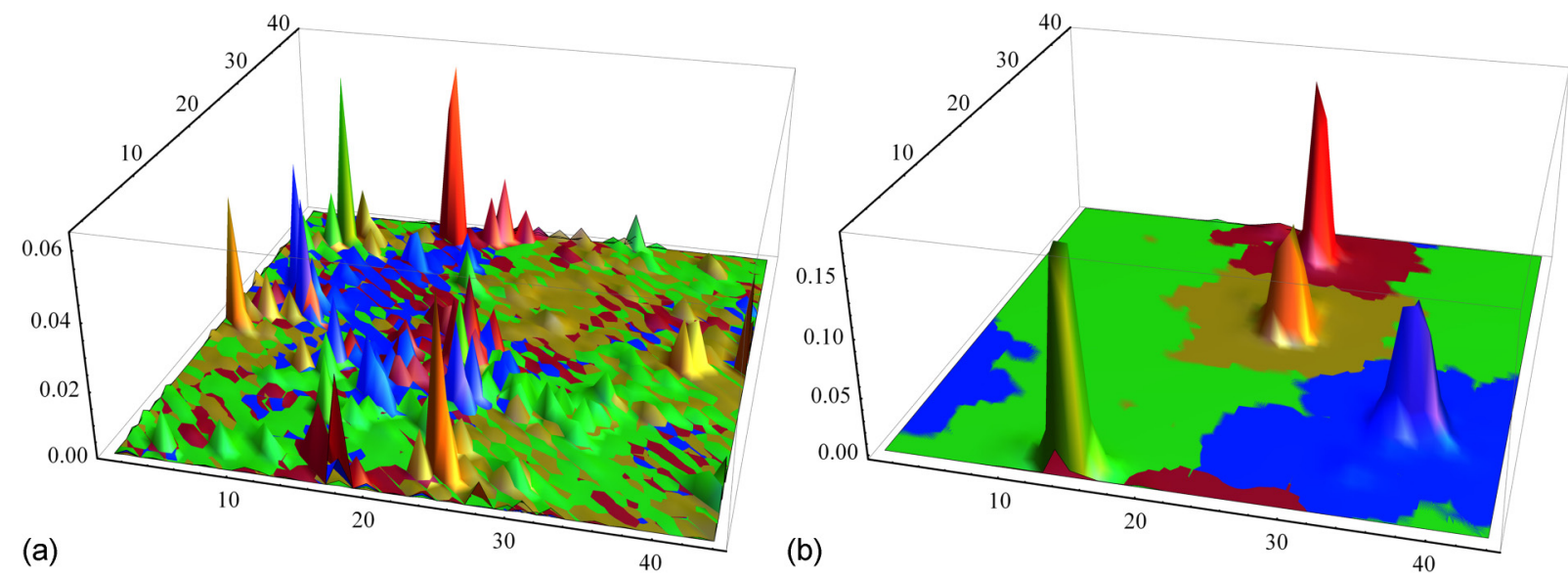

FIG. 1. (Color online) Multifractality, Chalker scaling, and interaction amplification. Critically delocalized (a) and Anderson localized (b) states. In each case we plot the probability density $\left|\psi_{n}(\mathbf{r})\right|^{2}$ for four different wave functions corresponding to four successive energy eigenvalues $\varepsilon_{n}<\varepsilon_{n+1}<\varepsilon_{n+2}<\varepsilon_{n+3}$ (blue, red, orange, green). Each critically delocalized state is multifractal [21-23] exhibiting an intricate inhomogeneous spatial structure. The structures of different wave functions with nearby energy eigenvalues are strongly correlated, a phenomenon called Chalker scaling [37-39]. This appears in (a) as the interweaving of different colored peaks to form calico "mountain ranges." The combination of multifractality and Chalker scaling can enhance short-ranged interparticle interactions [15,40,41]. By contrast, for the Anderson localized states in (b), there is essentially zero overlap between $\left|\psi_{n}(\mathbf{r})\right|^{2}$ and $\left|\psi_{m}(\mathbf{r})\right|^{2}$ for states with $0<\left|\varepsilon_{n}-\varepsilon_{m}\right| \ll \delta_{l}$, so that short-ranged interactions have negligible effect (at zero temperature) [42]. Here, $\delta_{l}$ denotes the level spacing in a characteristic localization volume. These wave functions were computed for a 2D class BDI lattice random hopping model [23], which is a nontopological analog for the CI surface states of a 3D topological superconductor [43].

using conformal field theory [15,19,20,24,32-36]. Our results for the class DIII multifractal spectra are new. In our previous work [15], we analyzed the effects of interactions on class CI TSCs, which possess spin SU(2) invariance. Here we do the same for classes AIII and DIII, which respectively possess $\mathrm{U}(1)$ and no spin symmetry. We enumerate four fermion interactions consistent with bulk symmetries, and compute the enhancement or suppression of interaction matrix elements due to the disorder. The combination of wave function multifractality [22,23] and dynamical Chalker scaling [37-39] can amplify interactions $[40,41]$ and even sabotage topological protection, as we found in class CI [15], see Fig. 1.

We show that in the limit of large topological winding numbers (many surface valleys), a weakly coupled sigma model allows a complimentary perturbative approach. We graft interparticle interactions into this framework, and thereby obtain Wess-Zumino-Novikov-Witten [45] Finkel'stein nonlinear sigma models $[46,47]$ (WZNW-FNLsMs) for all three classes. Using the exact conformal field theory results and one-loop WZNW-FNLsM renormalization group (RG) equations [15,48-51], we establish the restrictive conditions under which surface states can be robust to both disorder and interactions. For class DIII, we show that interactions are always irrelevant, while in class AIII, there is a finite window of stability, controlled by the disorder. Outside of this window we identify new interaction-stabilized fixed points.

We stress that here we focus on weak disorder and weak interactions. In particular, we consider only the effects of disorder at the surface, and we assume that the energy bandwidth of the microscopic disorder distribution is narrower than the bulk gap. Sufficiently strong surface disorder can induce a topologically trivial Anderson insulator in the outer layers of the crystal, submerging the nontrivial surface bands below [44]. Strong interactions at the surface can induce spontaneous time-reversal symmetry breaking, or realize gapped phases with topological order [52-54]. For modest disorder or interactions, it has been argued that the surface states of topological insulators and superconductors are protected [6]. We have shown that this is false in class CI, wherein we argued that the combination of arbitrarily weak disorder and interactions always induces spontaneous time-reversal symmetry breaking at surface [15].

Compared to analyzing the interplay of disorder and interactions in other Dirac materials $[55,56]$, the task of understanding topological superconductor surface states is much easier, due to the availability of nonperturbative techniques. The key tools employed in this work are conformal embeddings [45]. A conformal embedding defines systematic "fractionalization rules" for breaking up a level one affine Lie algebra (such as free fermions) into generalized "color" and "flavor" sectors. This is the non-Abelian version of spin-charge separation that applies, e.g., to $(1+1)$-D quantum chromodynamics [57]. For Dirac fermions coupled to quenched gauge fluctuations, the relevant embeddings are the three infinite two-parameter families [58]

\begin{tabular}{lccc}
\hline \hline Class & Embedding & $|v|$ & \\
\hline CI & $\mathrm{SO}(4 n k)_{1} \supset \mathrm{Sp}(2 n)_{k} \oplus \mathrm{Sp}(2 k)_{n}$ & $2 k$ & $(k \geqslant 1)$ \\
AIII & $\mathrm{U}(n k)_{1} \supset \mathrm{U}(n)_{k} \oplus \mathrm{SU}(k)_{n}$ & $k$ & $(k \geqslant 2)$ \\
DIII & $\mathrm{SO}(n k)_{1} \supset \mathrm{SO}(n)_{k} \oplus \mathrm{SO}(k)_{n}$ & $k$ & $(k \geqslant 3)$ \\
\hline \hline
\end{tabular}

Each family applies to a particular 3D TSC symmetry class. In this table, $|v|$ is the modulus of the bulk winding number, equal to the number of valleys ("colors"). For each embedding, this is specified by the parameter $k$, while the 
parameter $n$ plays the role of a replica ("flavor") index. The replica index is used to perform disorder averaging $[20,30,33,59]$. Identical results have been obtained for multifractal spectra in class AIII [32] and dynamic critical exponents in all three classes [36] using the supersymmetric [60] method; the corresponding embeddings appear in Ref. [36].

Three special cases do not fall into the scheme of Eq. (1.1). The minimal realization of class AIII has one surface valley $(|\nu|=1)$. In this case, time-reversal invariant perturbations couple only to the $U(1)$ spin current, in the form of an Abelian vector potential $[2,19,61]$. The minimal $|\nu|=1$ realization of class DIII admits no relevant or marginal time-reversal symmetric perturbations. The $|\nu|=2$ realization of class DIII is the same as the minimal $|\nu|=1$ version of AIII: a single complex Dirac fermion, perturbed by an Abelian $\mathrm{O}(2)$ vector potential.

We use the conformal embedding rules to obtain the effective conformal field theories relevant for computing disorder-averaged correlation functions in the noninteracting system. These are the level $k$ subalgebras in Eq. (1.1). In the many-valley $k \gg 1$ limit for each TSC class, the non-Abelian bosonization of the corresponding conformal theory becomes weakly coupled. This has exactly the same form as the nonlinear sigma model description of Anderson (de)localization expected in the appropriate symmetry class [23], except that it is augmented by a Wess-Zumino-Novikov-Witten term. The symmetry of the target manifold is $G \times G / G$, with $G \in$ $\{\mathrm{Sp}(2 n), \mathrm{U}(n), \mathrm{O}(n)\}$ for class CI, AIII, and DIII, respectively; $n$ is the number of replicas. For classes AIII and DIII (CI), the level $k$ of the Wess-Zumino-Novikov-Witten model is (half of) the bulk winding number modulus. This is also similar to the usual sigma model description of quantum diffusion, in that the coupling is proportional to the inverse universal Landauer conductance of the corresponding Dirac theory.

Unlike other applications of non-Abelian bosonization at higher levels $k>1$ (such as multicritical spin chains) [62], perturbations that break the global $G \times G$ invariance of the sigma model target manifold are forbidden. This is because the space of fluctuations (target manifold symmetry) is determined by the random matrix class [23] of the disordered Dirac Hamiltonian. To incorporate interactions, we need only interpret the target manifold as a product of replica times Matsubara frequency spaces. The allowed interactions can be inferred from the Dirac theory via the conformal embedding map, or written down directly in the sigma model based upon general considerations. We emphasize that the structure of the sigma model emerges directly from the conformal embedding and from a symmetry analysis of the $(2+1)$-D imaginary time path integral for the surface Majorana band [50,51]. We make no use of the self-consistent Born approximation or gradient expansion employed in diffusive metals $[59,60,63]$. We therefore obtain an interacting (Finkel'stein) [46,47] nonlinear sigma model without appealing to ideas of Fermi liquid theory.

The Majorana surface quasiparticles do not carry welldefined electric charge, but can conduct energy as well as spin, if the latter is conserved. In the absence of interactions, the zero temperature (Landauer) spin conductance in classes $\mathrm{CI}$ and AIII is unmodified by disorder, and assumes a finite universal value $[19,30,64]$. We argue in Sec. II B 2 that the same result applies to the thermal conductance (divided by the temperature) in class DIII. Numerical evidence $[61,65,66]$ suggests that the topology protects both wave function delocalization and strict conformal invariance in classes CI and AIII.

In a separate work [51], we establish that interactionmediated Altshuler-Aronov corrections to the spin or thermal conductance are suppressed in the conformal limit for all three TSC classes. This result obtains from the sigma models and is valid to lowest order in $1 / k$, but is nonperturbative in the interactions for classes CI and AIII. In Ref. [51], working directly with the disordered Majorana surface theory, we also prove that the lowest order Hartree and Fock corrections to the spin conductance vanish exactly for classes CI and AIII, in every fixed disorder realization. These results imply that the sigma model conductance parameter is pinned to the universal value at the conformal point in classes CI and AIII. Determining the stability of the phase then reduces to analyzing the $\mathrm{RG}$ equations for the interactions. We will conjecture that the same applies to class DIII, although the latter is stable for weak enough interactions regardless. The focus of the present paper is this stability analysis, and we also provide the technical details of our conformal field theory calculations.

The outline of this paper is as follows. In Sec. II, we present our results. We first transcribe low-energy field theories of TSC surface states in each class. We summarize the critical properties of noninteracting surface states in the presence of disorder. We then turn to the combined effects of disorder and interactions. We review previous results [15] in class CI, and present new results for AIII and DIII. We enumerate the interaction channels and scaling dimensions of the corresponding coupling strengths. We write down the one-loop RG equations for the corresponding WZNW-FNLsMs, valid in the large surface valley limit. Finally, we construct global phase diagrams as a function of the interaction coupling strengths.

In Sec. III, we derive the conformal field theory results discussed in Sec. II. The derivations of the one-loop RG equations for the WZNW-FNLsMs appear elsewhere [51]. We conclude with open questions in Sec. IV.

\section{MODELS AND RESULTS}

\section{A. Majorana surface bands for 3D topological superconductors}

Different from a $3 \mathrm{D} \mathbb{Z}_{2}$ topological insulator, the signature of a bulk topological superconductor (TSC) is the presence of any number of gapless, delocalized quasiparticle bands wrapping the sample surface. The absolute value of the bulk winding number [2] $|\nu|$ is equal to the number of independent species (or valleys) of surface quasiparticle bands.

In the periodic table $[2,3,5]$, there are three classes of topological superconductors in 3D denoted CI, AIII, and DIII. All are protected by time-reversal symmetry. The classes are distinguished by the degree of spin SU(2) symmetry enjoyed by the bulk and surface. Class CI requires full spin SU(2) invariance, even in the presence of disorder. A 3D class CI topological superconductor would require spin singlet pairing and a fully gapped bulk with negligible spin-orbit coupling. Class AIII is distinguished by spin U(1) invariance, as could be 
realized in a spin-triplet $p$-wave [50] TSC. Class DIII assumes no spin symmetry, e.g., a TSC with strong spin-orbit coupling. $\mathrm{Cu}_{x} \mathrm{Bi}_{2} \mathrm{Se}_{3}$ has been proposed as a possible realization of class DIII $[9,14,67-71]$. The $B$ phase of ${ }^{3} \mathrm{He}$ is a bulk topological superfluid in the same class $[2,16]$.

The form of the low-energy surface Dirac theory can be obtained in various ways. In Ref. [2], two general arguments were given. One is a topological field theory construction, while the second links the existence of a topological bulk in classes CI, AIII, and DIII to the "extended" random matrix classification [17] of Dirac fermions in 2D. A third way is to extract the surface states explicitly from a microscopic lattice model $[4,15]$. A derivation of this type for class AIII appears in Ref. [51]. The main output of this procedure is to verify the form of time-reversal symmetry, Eqs. (2.2) and (2.3).

\section{Spin $S U(2)$ symmetry: $C I$}

In class $\mathrm{CI}$, surface quasiparticle bands appear in valley degenerate pairs $[2,4]$. For a bulk with winding number $v=$ $\pm 2 k, k \in\{1,2, \ldots\}$, the low-energy Dirac theory can always be written as [15]

$$
H_{\mathrm{CI}}^{(0)}=\frac{1}{2 \pi} \int d^{2} \mathbf{r} \psi^{\dagger}\left\{\hat{\boldsymbol{\sigma}} \cdot\left[i \nabla-\mathbf{A}_{i}(\mathbf{r}) \hat{\mathfrak{t}}_{\kappa}^{i}\right]\right\} \psi .
$$

The complex Dirac field $\psi \rightarrow \psi_{\sigma, v, a}$ is a $4 n k$-component spinor, with indices in pseudospin $\sigma \in\{1,2\}$, valley $v \in$ $\{1,2, \ldots, 2 k\}$, and replica $a \in\{1,2, \ldots, n\}$ spaces. In this case, the pseudospin matrices $\hat{\sigma}^{1,2,3}$ act on a combination of Nambu (particle-hole) and orbital degrees of freedom, the precise interpretation of which requires a bulk microscopic model $[4,15]$. The vector operator $\hat{\sigma}=\left\{\hat{\sigma}^{1}, \hat{\sigma}^{2}\right\}$.

Time-reversal symmetry is encoded in the chiral transformation $[2,17]$

$$
\psi \rightarrow \hat{\sigma}^{3}\left(\psi^{\dagger}\right)^{\top}, \quad i \rightarrow-i,
$$

where $\mathrm{T}$ denotes the matrix transpose. This transformation is antiunitary and is equivalent to the condition

$$
-\hat{M}_{S} \hat{h} \hat{M}_{S}=\hat{h}, \quad \hat{M}_{S}=\hat{\sigma}^{3}
$$

for the single particle Hamiltonian $\hat{h}$ in $H_{\mathrm{CI}}^{(0)} \equiv \int \psi^{\dagger} \hat{h} \psi$.

That time-reversal invariance can be represented as a chiral symmetry is true for any Bogoliubov-de Gennes quasiparticle Hamiltonian (gapped or gapless, topological or trivial), and is a mere consequence of the particle-hole redundancy [50]. For 2D Dirac quasiparticles in classes CI, AIII, and DIII, only two unitarily inequivalent choices are possible for the conjugating matrix $\hat{M}_{S}$ [17]. In the topological case [Eqs. (2.2) and (2.3)], this restricts allowed perturbations to be off-diagonal in pseudospin space. As a consequence, only vector potentials are allowed [2]. By contrast, for 2D Dirac quasiparticles in topologically trivial class CI superconductors such as the $d$-wave cuprates, time-reversal symmetric perturbations include Dirac mass and scalar potentials terms [63]. In the latter case, $\hat{M}_{S}=\hat{\sigma}^{3} \hat{N}$, where $\hat{N}$ is a diagonal matrix in valley space with $p(q)$ elements equal to $+1(-1)$, $p, q \geqslant 1$, and $p+q=2 k$ ( $2 k$ is the number of valleys) [17]. The appearance of pseudomagnetic fields in a time-reversal invariant quasiparticle Hamiltonian is not a feature unique to topological superconductors. Rather, the special feature is the exclusion of other perturbations.
The vector potential $\mathbf{A}_{i}(\mathbf{r}) \hat{\mathfrak{t}}_{\kappa}^{i}$ in Eq. (2.1) encodes generic time-reversal symmetric surface potentials. These could include impurity charges or external electric fields, surface deformations of the bulk pairing strength, as well as edge, corner, dislocation or disclination potentials. The vector potential is non-Abelian, coupling the $2 k$ valleys through generators $\hat{\mathfrak{t}}_{\kappa}^{i} \rightarrow\left(\hat{\mathfrak{t}}_{\kappa}^{i}\right)_{v} v^{v^{\prime}}$ of the valley symmetry group $\operatorname{Sp}(2 k)$. Here, the lower (upper) index transforms in the fundamental (conjugate) representation. These are equivalent through raising or lowering by the symplectic "metric" $\left(\hat{\kappa}^{2}\right)^{v, v^{\prime}}=\left(\hat{\kappa}^{2}\right)_{v, v^{\prime}}$, which is a $2 k \times 2 k$ antisymmetric block Pauli matrix.

Spin SU(2) symmetry is hidden in Eq. (2.1); the U(1) charge of $\psi$ is the spin-projection along the $z$ axis (say). A spin rotation by $\pi$ around the $x$ axis appears as the unitary particlehole $(P)$ transformation

$$
\psi \rightarrow \hat{M}_{P}\left(\psi^{\dagger}\right)^{\top}, \hat{M}_{P}=i \hat{\sigma}^{1} \hat{\kappa}^{2} .
$$

Because $\hat{M}_{P}^{\top}=-\hat{M}_{P}$, we have $P^{2}=-1$ (class CI) $[2,3,5]$. Equation (2.4) restricts the non-Abelian vector potential in Eq. (2.1) to $\mathrm{Sp}(2 k)$ generators acting on valley space, since

$$
-\hat{\kappa}^{2}\left(\hat{\mathfrak{t}}_{\kappa}^{i}\right)^{\top} \hat{\kappa}^{2}=\hat{\mathfrak{t}}_{\kappa}^{i} .
$$

The Abelian Dirac 3-current encodes the $z$-spin density and associated spin current,

$$
\psi^{\dagger} \psi(\mathbf{r})=2 S^{z}(\mathbf{r}), \quad \psi^{\dagger} \hat{\boldsymbol{\sigma}} \psi(\mathbf{r})=2 \mathbf{J}^{z}(\mathbf{r}) .
$$

(Positive and negative spin densities are possible because the Dirac field has particle and antiparticle excitations.) The $z$-spin density (current) is odd (even) under time-reversal. The $x$ - and $y$-spin 3-currents are anomalous in the $\psi$ language, i.e., involve terms such as $\psi^{\top} \hat{\sigma}^{1} \hat{\kappa}^{2} \psi$ and $\psi^{\dagger} \hat{\sigma}^{1} \hat{\kappa}^{2}\left(\psi^{\dagger}\right)^{\top}$.

A manifestly covariant formulation obtains by defining

$$
\begin{aligned}
\left\{\mathcal{L}_{\uparrow, v, a}, \mathcal{L}_{\downarrow, v, a}\right\} & \equiv\left\{\psi_{1, v, a}, \psi_{2, a}^{\dagger v^{\prime}}\left(\hat{\kappa}^{2}\right)_{v^{\prime}, v}\right\}, \\
\left\{\mathcal{R}_{\uparrow, v, a}, \mathcal{R}_{\downarrow, v, a}\right\} & \equiv\left\{\psi_{2, v, a}, \psi_{1, a}^{\dagger v^{\prime}}\left(\hat{\kappa}^{2}\right)_{v^{\prime}, v}\right\}, \\
\mathrm{L} & \equiv \mathcal{L}^{\top} i \hat{s}^{2} \hat{\kappa}^{2} \rightarrow \mathrm{L}_{a}^{s, v}, \\
\mathrm{R} & \equiv \mathcal{R}^{\top} i \hat{s}^{2} \hat{\kappa}^{2} \rightarrow \mathrm{R}_{a}^{s, v} .
\end{aligned}
$$

The fields $\mathcal{L} \rightarrow \mathcal{L}_{s, v, a}$ and $\mathcal{R}_{s, v, a}$ transform in the fundamental representations of the spin $\mathrm{SU}(2)$ and valley $\mathrm{Sp}(2 k)$ symmetry groups. Here, $s \in\{\uparrow, \downarrow\}$ is the spin index. $L_{a}^{s, v}$ and $\mathrm{R}_{a}^{s, v}$ transform in the conjugate representations; $\hat{s}^{2}$ is the antisymmetric $2 \times 2$ Pauli matrix in spin space. All four fields $\left\{\mathcal{L}_{a}, \mathcal{R}_{a}, \mathrm{~L}_{a}, \mathrm{R}_{a}\right\}$ transform under the fundamental representation of $\mathrm{SO}(n)$ in replica space. Introducing complex coordinates $\{z, \bar{z}\}=x \pm i y$ and defining $\{\partial, \bar{\partial}\} \equiv \frac{1}{2}\left(\partial_{x} \mp i \partial_{y}\right)$, Eq. (2.1) can be rewritten as

$$
\begin{gathered}
H_{\mathrm{CI}}^{(0)}=H_{\mathrm{CI}}^{(0,1)}+H_{\mathrm{CI}}^{(0,2)} \\
H_{\mathrm{CI}}^{(0,1)}=-\frac{i}{2 \pi} \int d^{2} \mathbf{r}[\mathrm{L} \bar{\partial} \mathcal{L}+\mathrm{R} \partial \mathcal{R}], \\
H_{\mathrm{CI}}^{(0,2)}=\frac{1}{2 \pi} \int d^{2} \mathbf{r}\left[J_{\kappa}^{i} \bar{A}_{i}+\bar{J}_{\kappa}^{i} A_{i}\right] .
\end{gathered}
$$

In the last equation,

$$
\left\{A_{i}, \bar{A}_{i}\right\}=i\left(A_{i}^{x} \mp i A_{i}^{y}\right),
$$


TABLE I. Fierz and trace identities.

\begin{tabular}{lcc}
\hline \hline Algebra & Fierz identity & trace normalization \\
\hline $\mathrm{Sp}(2 k)$ & $\sum_{i}\left(\hat{\kappa}^{2} \hat{t}_{\kappa}^{i}\right)^{m n}\left(\hat{t}_{\kappa}^{i} \hat{\kappa}^{2}\right)^{p q}$ & $\operatorname{Tr}\left[\hat{t}_{\kappa}^{i} \hat{t}_{\kappa}^{j}\right]=\delta^{i j}$ \\
& $=\frac{1}{2}\left(\delta^{m q} \delta^{p n}+\delta^{m p} \delta^{q n}\right)$ & \\
$\mathrm{SU}(k)$ & $\sum_{i}\left(\hat{t}_{\kappa}^{i}\right)_{m}{ }^{n}\left(\hat{t}_{\kappa}^{i}\right)_{p}{ }^{q}$ & $\operatorname{Tr}\left[\hat{t}_{\kappa}^{i} \hat{t}_{\kappa}^{j}\right]=\delta^{i j}$ \\
& $=\delta_{m}{ }^{q} \delta_{p}{ }^{n}-\frac{1}{k} \delta_{m}{ }^{n} \delta_{p}{ }^{q}$ & \\
$\mathrm{SO}(k)$ & $\sum_{i}\left(\hat{t}_{\kappa}^{i}\right)^{m n}\left(\hat{t}_{\kappa}^{i}\right)^{p q}$ & $\operatorname{Tr}\left[\hat{t}_{\kappa}^{i} \hat{t}_{\kappa}^{j}\right]=2 \delta^{i j}$ \\
& $=\delta^{m q} \delta^{p n}-\delta^{m p} \delta^{q n}$ & \\
\hline \hline
\end{tabular}

and we have introduced the $\operatorname{Sp}(2 k)$ valley current operators

$$
\begin{aligned}
& J_{\kappa}^{i}(z) \equiv-\frac{i}{2} \mathrm{~L} \hat{\mathfrak{t}}_{\kappa}^{i} \mathcal{L}=\frac{1}{2} \mathcal{L}_{s, v, a}\left(\hat{s}^{2}\right)^{s, s^{\prime}}\left(\hat{\kappa}^{2} \hat{\mathfrak{t}}_{\kappa}^{i}\right)^{v, v^{\prime}} \mathcal{L}_{s^{\prime}, v^{\prime}, a}, \\
& \bar{J}_{\kappa}^{i}(\bar{z}) \equiv-\frac{i}{2} \mathrm{R} \hat{\mathfrak{t}}_{\kappa}^{i} \mathcal{R}=\frac{1}{2} \mathcal{R}_{s, v, a}\left(\hat{s}^{2}\right)^{s, s^{\prime}}\left(\hat{\kappa}^{2} \hat{\mathfrak{t}}_{\kappa}^{i}\right)^{v, v^{\prime}} \mathcal{R}_{s^{\prime}, v^{\prime}, a} .
\end{aligned}
$$

The current $J_{\kappa}^{i}\left[\bar{J}_{\kappa}^{i}\right]$ generates transformations in the holomorphic $\mathcal{L}(z)$ [antiholomorphic $\mathcal{R}(\bar{z})$ ] sector of the theory. Here and throughout the rest of this paper, doubly-repeated indices are summed unless otherwise indicated. Using Wick's theorem, one can check that the valley currents satisfy the $\mathrm{Sp}(2 k)_{n}$ Kac-Moody algebra:

$$
J_{\kappa}^{i}(z) J_{\kappa}^{j}(w) \sim \frac{n \delta^{i j}}{(z-w)^{2}}+\frac{i f_{\kappa}^{i j k}}{(z-w)} J_{\kappa}^{k}(w),
$$

where

$$
\left[\hat{\mathfrak{t}}_{\kappa}^{i}, \hat{\mathfrak{t}}_{\kappa}^{j}\right]=i f_{\kappa}^{i j k} \hat{\mathfrak{t}}_{\kappa}^{k} \text {. }
$$

The normalizations in Eqs. (2.10) and (2.11) reflect the trace and Fierz identities enumerated in Table I.

The fields $\mathcal{L}_{s, v, a}$ and $\mathcal{R}_{s, v, a}$ are each $4 n k$-component spinors. Equation (2.7b) is invariant under independent left and right $\mathrm{SO}(4 n k)$ transformations acting on the combined spin $\otimes$ valley $\otimes$ replica spaces:

$$
\begin{aligned}
\mathcal{L} & \rightarrow \hat{O} \mathcal{L}, \quad \mathrm{L} \rightarrow \mathrm{L} \hat{O}^{-1}, \\
\mathcal{R} & \rightarrow \hat{\bar{O}} \mathcal{R}, \quad \mathrm{R} \rightarrow \mathrm{R} \hat{\bar{O}}^{-1}, \\
\hat{O}^{-1} & =\hat{\sigma}^{2} \hat{\kappa}^{2} \hat{O}^{\top} \hat{\sigma}^{2} \hat{\kappa}^{2}, \quad \hat{\bar{O}}^{-1}=\hat{\sigma}^{2} \hat{\kappa}^{2} \hat{\bar{O}}^{\top} \hat{\sigma}^{2} \hat{\kappa}^{2} .
\end{aligned}
$$

We can replace the Hamiltonian with a $(2+0)$-D Grassmann path integral; then the clean theory with $A_{i}=\bar{A}_{i}=0$ is equivalent to the $\mathrm{SO}(4 n k)_{1}$ conformal field theory. The class CI embedding scheme in Eq. (1.1) implies that this theory can be decomposed into $\operatorname{Sp}(2 k)_{n}$ valley and $\operatorname{Sp}(2 n)_{k}$ spin $\otimes$ replica sectors.

\section{Spin U(1) symmetry: AIII}

The winding number is integer-valued for class AIII, so that the surface quasiparticle action for a TSC can have any number of $k \in\{1,2, \ldots\}$ valleys. Time-reversal symmetric surface perturbations are non-Abelian and Abelian vector potentials that couple to the valley and spin U(1) currents, respectively. The minimal case of $k=1$ is special, in that only the Abelian spin current can appear. The latter model has been extensively studied [19,24-29,65,66]. The non-Abelian case was analyzed in Refs. [20,30-35,64].

The Hamiltonian for an AIII TSC with winding number $k>1$ is

$$
H_{\mathrm{AIII}}^{(0)}=\frac{1}{4 \pi} \int d^{2} \mathbf{r} \psi^{\dagger}\left\{\hat{\boldsymbol{\sigma}} \cdot\left[i \nabla-\mathbf{A}_{i}(\mathbf{r}) \hat{\mathfrak{t}}_{\kappa}^{i}-\mathbf{A}(\mathbf{r})\right]\right\} \psi .
$$

The complex field $\psi$ is interpreted the same way as in class CI, except that the valley index $v \in\{1,2, \ldots, k\}$. The $\mathrm{U}(1)$ charge corresponds to the conserved $z$-spin density. The Abelian vector potential A couples to the corresponding current [Eq. (2.5)]. The non-Abelian potential $\mathbf{A}_{i}$ couples to $\mathrm{SU}(k)$ generators $\hat{\mathfrak{t}}_{\kappa}^{i}$ acting on valley space.

We define the pseudospin decomposition,

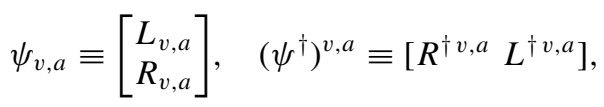

which leads to

$$
\begin{gathered}
H_{\mathrm{AIII}}^{(0)}=H_{\mathrm{AIII}}^{(0,1)}+H_{\mathrm{AIII}}^{(0,2)}, \\
H_{\mathrm{AIII}}^{(0,1)}=\frac{i}{2 \pi} \int d^{2} \mathbf{r}\left[L^{\dagger} \bar{\partial} L+R^{\dagger} \partial R\right], \\
H_{\mathrm{AIII}}^{(0,2)}=\frac{1}{2 \pi} \int d^{2} \mathbf{r}\left[J_{\kappa}^{i} \bar{A}_{i}+\bar{J}_{\kappa}^{i} A_{i}+J \bar{A}+\bar{J} A\right] .
\end{gathered}
$$

In the last equation, the vector potential components are defined as in Eq. (2.8), while

$$
\begin{aligned}
& J_{\kappa}^{i}(z)=-i \gamma_{\kappa} L^{\dagger v, a}\left(\hat{\mathfrak{t}}_{\kappa}^{i}\right)_{v}^{v^{\prime}} L_{v^{\prime}, a}, \\
& J(z)=-i \gamma_{0} L^{\dagger v, a} L_{v, a} .
\end{aligned}
$$

The constants $\gamma_{\kappa, 0}$ are chosen so that $J_{\kappa}^{i}(z)[J(z)]$ satisfies the canonical SU $(k)_{n}[\mathrm{U}(1)]$ Kac-Moody algebra.

Equation (2.15b) is invariant under $\mathrm{U}(n k) \times \mathrm{U}(n k)$ replica $\otimes$ valley $\otimes$ spin $U(1)$ transformations, and is equivalent to $\mathrm{U}(n k)_{1}$. The AIII embedding scheme in Eq. (1.1) implies that this can be decomposed into $\mathrm{SU}(k)_{n}$ valley and spin $\mathrm{U}(1) \otimes$ replica $\mathrm{SU}(n)_{k}$ sectors $[20,30,33]$.

\section{No spin symmetry: DIII}

In class DIII, the winding number $v \in \mathbb{Z}$ and there are $k=|v|$ surface quasiparticle bands. Spin SU(2) invariance is completely destroyed, as occurs in the presence of strong spin-orbit coupling. Another way to realize class DIII surface states is to deposit strong spin-orbit scattering impurities on the surface of an erstwhile class CI or AIII TSC.

Because spin is not conserved, there is no natural $\mathrm{U}(1)$ with which to define complex Dirac spinors for the surface quasiparticle bands. Instead, we are forced to work with a real Majorana spinor $\chi_{\sigma, v, a}$, with pseudospin ( $\sim$ real spin) index $\sigma \in\{1,2\}$, valley $v \in\{1,2, \ldots, k\}$, and replica $a \in$ $\{1,2, \ldots, n\}$. The $k=1$ case admits no relevant or marginal perturbations, so (weak) disorder has a negligible effect. The $k=2$ case is identical to AIII with $k=1$, a single complex Dirac fermion coupled to an Abelian vector potential. In this case the $\mathrm{U}(1)$ charge is the valley polarization. 
For $k \geqslant 3$ (three or more valleys), DIII admits non-Abelian intervalley scattering. The Hamiltonian is

$$
H_{\mathrm{DIII}}^{(0)}=\frac{1}{4 \pi} \int d^{2} \mathbf{r} \chi^{\top} \hat{M}_{P}\left\{\hat{\boldsymbol{\sigma}} \cdot\left[i \boldsymbol{\nabla}-\mathbf{A}_{i}(\mathbf{r}) \hat{\mathfrak{t}}_{\kappa}^{i}\right]\right\} \chi,
$$

where

$$
\hat{M}_{P}=-i \hat{\sigma}^{1}
$$

is a particle-hole conjugation matrix. $\left(\hat{M}_{P}^{\top}=\hat{M}_{P}\right.$, so that $P^{2}=+1$, class DIII.) Time reversal $(T)$ is encoded by the transformation

$$
\chi \rightarrow \hat{M}_{T} \chi, \quad i \rightarrow-i, \quad \hat{M}_{T}=-i \hat{\sigma}^{2},
$$

which implies that $T^{2}=-1$. The vector potential couples to valley space generators $\left\{\hat{\mathfrak{t}}_{\kappa}^{i}\right\}$ for $\mathrm{SO}(k)$, since these must satisfy

$$
-\left(\hat{\mathfrak{t}}_{\kappa}^{i}\right)^{\top}=\hat{\mathfrak{t}}_{\kappa}^{i} .
$$

With the decomposition

$$
\chi_{v, a} \equiv\left[\begin{array}{l}
L_{v, a} \\
R_{v, a}
\end{array}\right]
$$

the Hamiltonian can be rewritten as

$$
\begin{aligned}
H_{\mathrm{DIII}}^{(0)} & =H_{\mathrm{DIII}}^{(0,1)}+H_{\mathrm{DIII}}^{(0,2)}, \\
H_{\mathrm{DIII}}^{(0,1)} & =\frac{1}{2 \pi} \int d^{2} \mathbf{r}[L \bar{\partial} L+R \partial R], \\
H_{\mathrm{DIII}}^{(0,2)} & =\frac{1}{2 \pi} \int d^{2} \mathbf{r}\left[J_{\kappa}^{i} \bar{A}_{i}+\bar{J}_{\kappa}^{i} A_{i}\right] .
\end{aligned}
$$

The valley currents $J_{\kappa}^{i}(z)=-i \gamma_{k} L^{\top} \hat{\mathfrak{t}}_{\kappa}^{i} L$ in Eq. (2.21c) generate an $\mathrm{SO}(k)_{n}$ Kac-Moody algebra. The free Hamiltonian in Eq. (2.21b) is invariant under $\mathrm{SO}(n k) \times \mathrm{SO}(n k)$ replica $\otimes$ valley space transformations, and is equivalent to $S O(n k)_{1}$. The DIII embedding in Eq. (1.1) implies that this can be decomposed into $\mathrm{SO}(k)_{n}$ valley and $\mathrm{SO}(n)_{k}$ replica sectors.

\section{Spin and thermal quantum Hall effects: Dirac mass order parameters for imaginary surface state Cooper pairing}

For each of the three classes of TSCs there is a single valley-invariant Dirac surface mass operator:

$$
\begin{array}{ll}
m(\mathbf{r})=\psi^{\dagger} \hat{\sigma}^{3} \psi, & \text { CI and AIII [Eqs. (2.1), (2.13)] } \\
m(\mathbf{r})=\chi^{\top} \hat{M}_{P} \hat{\sigma}^{3} \chi, & \text { DIII [Eq. (2.17)]. }
\end{array}
$$

In each case, this operator breaks time-reversal invariance and opens up a gap in the surface spectrum at the Dirac point, but preserves spin SU(2) [U(1)] symmetry in class CI (AIII).

In fact, a Dirac mass $\langle m\rangle \neq 0$ induces a surface quantum Hall effect, analogous to the "half-integer" quantum Hall phase at the surface of a $3 \mathrm{D} \mathbb{Z}_{2}$ topological insulator with broken time-reversal symmetry $[6,72,73]$. In each case, the mass modifies the surface state symmetry class. Class CI shifts to class $\mathrm{C}$; the gapped surface resides in a plateau of the SU(2) invariant spin quantum Hall effect $[4,74]$. This state is characterized by a quantized spin Hall conductance $[15,74]$

$$
\sigma_{x y}^{s}=\frac{1}{h}\left(\frac{\hbar}{2}\right)^{2} k \operatorname{sgn}\langle m\rangle,
$$

where $k=|v| / 2$ is half the number of surface valleys, and $\hbar / 2$ is the "spin charge." The spin Hall current could be induced on a closed TSC surface divided into two complimentary domains $A$ and $B$, with $\langle m\rangle>0(\langle m\rangle<0)$ in domain $A(B)$. For $k=1$, the domains would be separated by a one-dimensional, two-channel edge state (corresponding to up and down spins). Applying a linearly varying Zeeman field across domain $A$ (say) would induce a circulating spin edge current with polarization equal to that of the external field. The physics is similar in class AIII, which shifts to class A. The gapped phase is a plateau of the "ordinary" quantum Hall effect, with the conserved $z$ spin taking the place of electric charge.

A topological superconductor in class DIII conserves only heat, so a mass term induces a thermal quantum Hall state. Majorana edge states separating spatial domains in different plateaux demonstrate a quantized thermal Hall conductivity $[72,73,75]$ in the presence of a transverse temperature gradient.

How can a nonzero Dirac mass be induced? For a TSC, it turns out that $\langle m\rangle$ can be viewed as the order parameter amplitude for imaginary Cooper pairing of the surface Majorana quasiparticles [15]. In other words, due to attractive interactions the gapless surface quasiparticles can Cooper pair at a nonzero superfluid phase angle relative to the bulk. This is necessary to open a gap and break time-reversal symmetry; pairing of the surface at the same angle as the bulk would appear as a vector potential order parameter, which neither opens the gap nor localizes the surface quasiparticles. Indeed, a homogeneous time-reversal invariant pairing merely shifts the surface Dirac points, and can be removed from the low energy theory by a gauge transformation.

For a clean TSC surface, short-ranged interactions are strongly irrelevant, and Cooper pairing is only possible for relatively strong interactions. However, disorder can enhance interaction effects, due to the strong spatial inhomogeneities induced in delocalized wave functions by quantum interference $[15,40,41,76]$. In class CI, it turns out interactions have a strong effect for arbitrarily weak disorder, due to the enhancement of interaction matrix elements by multifractal fluctuations in the local density of states (Ref. [15]; see also Sec. II D 1, below). For any winding number $|v|=2 k \neq 0$ in class CI, time-reversal breaks spontaneously at the surface. The dominant interaction channel favors $\langle m\rangle \neq 0$.

One can define other types of Dirac mass operators that do not preserve valley symmetry. These can serve as order parameters for states with valley space symmetry that is broken on average (after summing over disorder realizations). The noninvariant mass operators also break time-reversal symmetry. Tuning the amplitudes of these relative to $\langle m\rangle$, one can drive the surface across plateau transitions into different surface Hall states. Mass operators of this type in class CI are discussed in Appendix A. Dirac mass operators appear again in Sec. II D, wherein we discuss surface interaction channels and instabilities.

\section{B. Critical delocalization: noninteracting properties}

In this section, we describe the influence of disorder on noninteracting topological superconductor surface states. The objects of study are the density of states exponents and 
multifractal spectrum defined below via Eqs. (2.28) and (2.33), respectively. Exact conformal field theory results for all three classes appear in Eqs. (2.30) and (2.36), most of which were previously known. In Sec. II B 2, we address the stability of these effective theories in the absence of interactions and discuss evidence that topological protection extends to strict conformal invariance.

We briefly summarize the logic of the approach. Technical details are relegated to Sec. III. To treat the influence of disorder, we can write a $(2+0)$-D Grassmann path integral with the action given by Eqs. (2.7), (2.15), or (2.21) for the appropriate symmetry class. One can view this as the zero Matsubara frequency component of an imaginary time formulation. Without interactions, different frequencies decouple. The strong correlations between any two (nearly) delocalized wave functions as a function of their energy separation is known as Chalker scaling [37-39,61] and is a universal characteristic of disorder-mediated quantum interference. Chalker scaling implies that low-energy properties can be extracted from the dimensions of local operators in the effective zero energy theory. These include the scaling behavior of the density of states with energy [19,20] and the static enhancement or suppression of interaction matrix elements due to the disorder $[15,40,41,76]$. We use replicas to average over disorder configurations [59], with the number of replicas $n \rightarrow 0$ at the end.

For any amount of non-Abelian valley disorder on the surface of a 3D topological superconductor, the description in terms of the clean Dirac band structure is unstable in the sense of the renormalization group (RG) [20]. For example, consider the Gaussian white noise correlated disorder,

$$
\overline{A_{i}^{\alpha}(\mathbf{r}) A_{j}^{\beta}\left(\mathbf{r}^{\prime}\right)}=\lambda_{N A} \delta_{i j} \delta^{\alpha \beta} \delta^{(2)}\left(\mathbf{r}-\mathbf{r}^{\prime}\right),
$$

where the overline denotes an ensemble average over disorder realizations, and $\lambda_{N A}>0$ is the disorder variance. Using the Kac-Moody operator product expansion for the valley currents as in Eq. (2.10), the one-loop renormalization group (RG) equation is [77]

$$
\frac{d \lambda_{N A}}{d l}=2 \pi g \lambda_{N A}^{2}+\boldsymbol{O}\left(\lambda_{N A}\right)^{3} .
$$

Here, $g$ is the dual Coxeter number (half the quadratic Casimir in the adjoint representation) for the valley symmetry group [45]

$$
\begin{aligned}
g[\operatorname{Sp}(2 k)] & =k+1, \\
g[\operatorname{SU}(k)] & =k, \\
g[\operatorname{SO}(k)] & =k-2 .
\end{aligned}
$$

As discussed below Eq. (1.1), $k \geqslant 2(k \geqslant 3)$ for class AIII (DIII), so that Eq. (2.25) implies that the effects of disorder grow stronger as we renormalize towards longer length scales and lower energies.

A diverging RG flow implies that the correct effective field theory is strongly coupled relative to the free field (clean Dirac) fixed point. Note, however, that the non-Abelian disorder couples only to the valley Kac-Moody currents in each of Eqs. (2.7c), (2.15c), and (2.21c).

The conformal embeddings in Eq. (1.1) imply that the clean theory in Eqs. (2.7b), (2.15b), and (2.21b) can be decomposed into the "sum" of the level $n$ valley algebra $\left[\equiv G(k)_{n}\right]$, and the level $k$ replica or replica $\otimes$ spin algebra $\left[\equiv H(n)_{k}\right]$. What this means is that the holomorphic stress tensor $T(z)$ for the clean theory can be written as the sum [45]

$$
T(z)=T_{n}^{[G(k)]}(z)+T_{k}^{[H(n)]}(z),
$$

where

$$
T_{q}^{[G]}(z)=\frac{1}{2(q+g)}: J^{\alpha} J^{\alpha}:
$$

is the Sugawara stress tensor for the level $q$ algebra $G_{q}$ ( $g$ is the dual Coxeter number). Roughly speaking (but see, e.g., Ref. [57]), the full operator content of the free level one theory in each embedding of Eq. (1.1) can be decomposed into Kac-Moody currents of the $G(k)_{n}$ and $H(n)_{k}$ subalgebras, or products of primary and/or descendant fields from these two sectors.

Because the disorder is a relevant perturbation in the sense of Eq. (2.25), yet couples only to the valley algebra $G(k)_{n}$ in each symmetry class, the effective low-energy, long-wavelength theory of the disorder-averaged TSC surface state is the "conformal remnant" $H(n)_{k}$. That is, the conformal field theory that one can use to calculate disorder-averaged correlation functions is $\mathrm{Sp}(2 n)_{k}$ in class CI [4,15], $\mathrm{U}(n)_{k}$ in class AIII [20,30], and $\mathrm{SO}(n)_{k}$ in class DIII. An alternative scheme based upon supersymmetric embeddings [32,36] gives identical results.

Note that the effective level- $k$ theories know nothing about the microscopic character of the non-Abelian impurity potential. Indeed, the main effect of the RG flow in Eq. (2.25) is to "gauge away" the disorder in valley space, leaving behind the replica algebra with which we perform subsequent calculations. A similar phenomenon occurs in $(1+1)-\mathrm{D}$ QCD, wherein the color space becomes massive and decouples from a conformally invariant flavor remnant [57]. As a result, the predictions for disorder-averaged correlation functions are universal for classes CI and DIII. Class AIII admits an additional disorder parameter $\lambda_{A}$ [Eq. (2.31), below], which encodes the strength of vector potential fluctuations that couple to the Abelian spin U(1) current. The predictions for class AIII are therefore one-parameter functions of this (see below).

We stress that for non-Abelian disorder, it is not necessary to assume the Gaussian white noise correlations in Eq. (2.24). In general, white noise is a suitable replacement for any disorder potential that is sufficiently short-range correlated. (The criterion is that momentum space correlation function must exist as $\mathbf{k} \rightarrow 0$.) For TSC surface states, Coulomb impurities should be well-screened by the bulk superfluid, but other sources of long-range correlated disorder could exist [78]. Long-range correlations produce a more strongly divergent RG flow [78], but we expect the same universal description of the surface states described above (with the exception of long-range correlated Abelian disorder in class AIII).

\section{Density of states and multifractal spectra}

The average density of states $v(\varepsilon)$ is critical for Dirac fermions coupled to vector potential disorder [19,20,2730]. The scaling behavior with respect to the energy $\varepsilon$ is 
determined by

$$
v(\varepsilon) \sim|\varepsilon|^{x_{1} / z} .
$$

Here, all energies are measured relative to the surface state Dirac point, taken to reside at $\varepsilon=0$. In Eq. (2.28), $x_{1}$ is the scaling dimension of the disorder-averaged local density of states $(\mathrm{LDoS})$ operator $\equiv \overline{v(\varepsilon, \mathbf{r})}[79]$, and $z$ is the dynamic critical exponent. These are related by

$$
2=z+x_{1} .
$$

For the three classes of disordered TSCs, one finds that

\begin{tabular}{lll}
\hline$\overline{\text { Class }}$ & $|v|$ & $x_{1} / z$ \\
\hline CI & $2 k$ & $\frac{1}{4 k+3}$ \\
AIII & $k \geqslant 1$ & $\frac{1-k^{2} \lambda_{A}}{2 k^{2}-1+\lambda_{A} k^{2}}$ \\
DIII & $k \geqslant 3$ & $-\frac{1}{2 k-3}$ \\
\hline \hline
\end{tabular}

All of the results in Eq. (2.30) were previously known. The result for AIII with $\lambda_{A}=0$ first appeared in Ref. [20]. Here, $\lambda_{A}$ is the strength of the Abelian vector potential disorder in Eqs. (2.13) and (2.15c):

$$
\overline{A^{\alpha}(\mathbf{r}) A^{\beta}\left(\mathbf{r}^{\prime}\right)}=\lambda_{A} \delta^{\alpha \beta} \delta^{(2)}\left(\mathbf{r}-\mathbf{r}^{\prime}\right) .
$$

The parameter $\lambda_{A}$ is strictly marginal in the conformal limit, and can be thought of as an effective "Luttinger parameter" in the spin U(1) sector. For a single valley $k=1$, the AIII result above reproduces that of Ref. [19], which first considered Abelian disorder. The full expression for $x_{1} / z$ was implicit in Ref. [32]. Results for CI and DIII first appeared in Ref. [36].

For class CI, the average density of states always vanishes as $\varepsilon \rightarrow 0$, but the power approaches zero as $k \rightarrow \infty$. The same is true for class AIII for $\lambda_{A}<1 / k^{2}$, while divergent behavior obtains in the opposite case. The average density of states for DIII is always divergent at zero energy. Similar behavior was found in the class DIII nonlinear sigma model without the Wess-Zumino-Novikov-Witten term [80]. In this paper, we only discuss the regime of weak Abelian disorder $\lambda_{A}<2+\frac{1-k}{k^{2}}$, so that we avoid the "freezing" transition that occurs in the density of states [2729,61] and multifractal spectrum [24-26,61] at stronger disorder

The disorder-induced spatial fluctuations of the LDoS $\nu(\varepsilon, \mathbf{r})$ are encoded in the multifractal spectrum [21-23] $\tau(q)$. The $\tau(q)$ spectrum measures the sensitivity of extended wave functions to the sample boundary. A large $L \times L$ area of the surface is finely partitioned into a grid of boxes of size $a \ll L$. One then defines the box probability $\mu_{n}$ and inverse participation ratio $\mathcal{P}_{q}$ in terms of a typical wave function $\psi(\mathbf{r})$ :

$$
\mu_{n} \equiv \frac{\int_{\mathcal{A}_{n}} d^{2} \mathbf{r}|\psi(\mathbf{r})|^{2}}{\int_{L^{2}} d^{2} \mathbf{r}|\psi(\mathbf{r})|^{2}}, \quad \mathcal{P}_{q} \equiv \sum_{n} \mu_{n}^{q},
$$

where $\mathcal{A}_{n}$ denotes the $n^{\text {th }}$ box. The normalization is such that $\mathcal{P}_{1}=1$.
For a critically delocalized eigenstate $\psi$ (such as that found at a mobility edge),

$$
\mathcal{P}_{q} \sim\left(\frac{a}{L}\right)^{\tau(q)},
$$

where the exponent $\tau(q)$ is self-averaging and typically universal [24]. The multifractal spectrum thus provides a unique fingerprint for spatial fluctuations in a particular symmetry class. In the field-theoretic description, the $q$ th moment of the disorder-averaged $\operatorname{LDoS}(q \in\{1,2,3 \ldots\})$ is associated to a particular composite operator $\mathcal{O}_{q}$, with scaling dimension $x_{q}$. The set of such dimensions determines the multifractal spectrum via

$$
\tau(q)=2(q-1)+x_{q}-q x_{1} .
$$

By contrast, localized states are insensitive to the sample boundary for sufficiently large $L$ and have $\tau(q)=0$.

For topological superconductor surface states, the multifractal spectrum $\tau(q)$ is exactly quadratic:

$$
\tau(q)=(q-1)\left(2-\theta_{k} q\right),
$$

where

\begin{tabular}{lll}
\hline$\overline{\text { Class }}$ & $|v|$ & $\theta_{k}$ \\
\hline CI & $2 k$ & $\frac{1}{2(k+1)}$ \\
AIII & $k \geqslant 1$ & $\frac{k-1}{k^{2}}+\lambda_{A}$ \\
DIII & $k \geqslant 3$ & $\frac{1}{k-2}$ \\
\hline \hline
\end{tabular}

The results for CI and AIII were first obtained in Refs. [15] and [32,33], respectively. The DIII result is new. The class AIII result for $k=1$ is that expected for a single Dirac fermion subject to Abelian vector potential disorder [19]. \{In fact, Eq. (2.35) holds only for $|q| \leqslant q_{c}$, with $q_{c}=\sqrt{2 / \theta_{k}}$. Outside of this region the spectrum is linear ("termination") [2326,81].\}

The associated scaling exponents $\left\{x_{q}\right\}$ satisfy the symmetry relation [82-84]

$$
x_{q}=x_{q_{*}-q},
$$

with $q_{*}=2$ for class CI [83] and $q_{*}=0$ for class DIII [84], see Ref. [85].

Wave function multifractality plays the major role in our discussion of interaction effects and the stability of topological superconductor surface phases [Sec. II C, below]. In addition, multifractal spectra can be extracted from real space LDoS data (obtained, e.g., via STM) [86-88].

\section{Topological protection of conformal invariance}

The exact results in Eqs. (2.30) and (2.36) obtain for the conformally invariant level $k$ subalgebras in Eq. (1.1). We have argued that these emerge through "fractionalization" of the disordered Dirac theories in the low-energy, long-wavelength limit. The mechanism involves the conformal embedding schemes in Eq. (1.1). However, the precise character of the RG flow connecting the perturbed Dirac theory to the effective 
level $k$ conformal field theory (CFT) is not clear. In principle, we should consider perturbations away from the conformal fixed points, in order to determine their stability. Here, we focus exclusively on the noninteracting theory; interactions are treated in the next section.

One crucial difference between the level $k$ CFTs employed here, as compared to other applications of non-Abelian bosonization, is that perturbations which break global $G \times G$ invariance of the corresponding sigma model target manifold are disallowed. The action for an affine Lie algebra $G_{k}$ is [45]

$$
S_{G}^{(k)}(\lambda)=\frac{1}{\lambda} \int_{\partial B} \frac{d^{2} \mathbf{r}}{8 \pi l_{\phi}} \operatorname{Tr}\left(\partial_{\mu} \hat{Q}^{\dagger} \partial_{\mu} \hat{Q}\right)+k S_{\mathrm{WZNW}},
$$

where the Wess-Zumino-Novikov-Witten term is defined via

$$
\begin{aligned}
S_{\mathrm{WZNW}}= & -i \int_{B} \frac{d^{3} \mathbf{y}}{12 \pi l_{\phi}} \epsilon^{\alpha \beta \gamma} \\
& \times \operatorname{Tr}\left[\left(\hat{Q}^{\dagger} \partial_{\alpha} \hat{Q}\right)\left(\hat{Q}^{\dagger} \partial_{\beta} \hat{Q}\right)\left(\hat{Q}^{\dagger} \partial_{\gamma} \hat{Q}\right)\right] .
\end{aligned}
$$

Here, $B$ denotes a 3 -manifold with $2 \mathrm{D}$ boundary $\partial B$. The former (latter) can be taken as the interior volume (closed surface) of the TSC [89]. The parameter $l_{\phi}$ is the Dynkin index of the corresponding group. The matrix field $\hat{Q}(\mathbf{r})$ takes values in the compact Lie group $G$. Equations (2.38) and (2.39) are manifestly invariant under the $G \times G$ transformation

$$
\hat{Q}(\mathbf{r}) \rightarrow \hat{U}_{L} \hat{Q}(\mathbf{r}) \hat{U}_{R},
$$

where $U_{L, R}$ are independent, unitary global transformations in $G$. At the conformal fixed point [90], the coupling strength $\lambda$ of the gradient term in Eq. (2.38) is equal to $1 / k$.

Typically, global $G \times G$ invariance is an emergent property [62] of a 2D conformal field theory, not present in a parent microscopic model. By contrast, Eqs. (2.7), (2.15), and (2.21) are invariant under $H(n) \otimes H(n)$ left and right (holomorphic and antiholomorphic) group transformations, where $H(n) \in\{\mathrm{Sp}(2 n), \mathrm{U}(n), \mathrm{O}(n)\}$ for classes CI, AIII, and DIII, respectively. This result is more general than the conformal invariance itself. It applies to the replicated Grassmann path integration encoding correlation functions of any $d$ dimensional single particle Hamiltonian in the associated symmetry class $[23,50,51]$. Thus we cannot add "mass" terms such as $\operatorname{Tr}\left(\hat{Q}+\hat{Q}^{\dagger}\right)$ or $\operatorname{Tr}(\hat{Q}) \operatorname{Tr}\left(\hat{Q}^{\dagger}\right)$ to Eq. (2.38).

The CFT is distinguished by local $G \times G$ invariance, which is not a property of the Dirac theories in Eqs. (2.1), (2.13), and (2.17) for nonzero disorder. Tuning the gradient coupling $\lambda$ in Eq. (2.38) away from $1 / k$ preserves global but not local invariance. Perturbing around the CFT, the lowest-order RG equation for the deformation

$$
\delta \lambda \equiv \lambda-\frac{1}{k}
$$

is given by

$$
\frac{d \ln \delta \lambda}{d l}=-\frac{2 g}{k+g}+\boldsymbol{O}(\delta \lambda),
$$

where $k$ is the level and $g$ is the dual Coxeter number. For the level $k$ algebras in Eq. (1.1) corresponding to the TSC classes, in the replica $n \rightarrow 0$ limit, we have

$$
\begin{array}{lll}
\text { CI } \quad\left[\operatorname{Sp}(2 n)_{k}\right]: & \frac{d \ln \delta \lambda}{d l}=-\frac{2}{(k+1)}, \\
\text { AIII } \quad\left[\operatorname{SU}(n)_{k}\right]: & \frac{d \ln \delta \lambda}{d l}=0, \\
\text { DIII } \quad\left[\mathrm{SO}(n)_{k}\right]: & \frac{d \ln \delta \lambda}{d l}=\frac{4}{(k-2)},
\end{array}
$$

up to corrections of order $\delta \lambda$. In the large- $k$ limit, the sigma model description with $\lambda \sim 1 / k$ in Eq. (2.38) becomes weakly coupled. Perturbing around the Gaussian fixed point $[91](\lambda=$ $0)$ gives the complimentary flow equations:

$$
\begin{array}{lll}
\text { CI } \quad\left[\operatorname{Sp}(2 n)_{k}\right]: & \frac{d \lambda}{d l}=\lambda^{2}\left[1-(k \lambda)^{2}\right], \\
\text { AIII } \quad\left[\operatorname{SU}(n)_{k}\right]: & \frac{d \lambda}{d l}=0, \\
\text { DIII } & {\left[\operatorname{SO}(n)_{k}\right]:} & \frac{d \lambda}{d l}=-2 \lambda^{2}\left[1-(k \lambda)^{2}\right],
\end{array}
$$

up to corrections of order $\lambda^{3} \sim 1 / k^{3}$. Linearizing about the nontrivial fixed point in each case, Eqs. (2.41a)-(2.41c) are consistent with (2.40) to lowest order in $1 / k$.

For class AIII, we must also consider the renormalization of the $U(1)$ disorder strength $\lambda_{A}$. This is given by [92]

$$
\text { AIII : } \frac{d \lambda_{A}}{d l}=\lambda^{2}\left[1-(k \lambda)^{2}\right] .
$$

We derive Eq. (2.42) in Appendix B.

We consider each TSC class in turn. Equations (2.40a) and (2.41a) imply that the class CI CFT is an attractive fixed point, in the absence of interactions. We therefore expect the disordered Dirac theory [Eq. (2.1)] to flow into the $\operatorname{Sp}(2 n)_{k}$ fixed point in the long wavelength limit [15]. By contrast, the class DIII CFT is unstable according to Eqs. (2.40c) and (2.41c) (recall that $k \geqslant 3$ for class DIII). Class DIII is known to possess a stable metallic phase in 2D [80].

Class AIII presents a particularly peculiar case: the parameter $\lambda$ is marginal [Eqs. (2.40b) and (2.41b)], but the Abelian disorder strength $\lambda_{A}$ flows to strong coupling for $\lambda \neq 1 / k$ [Eq. (2.42)]. Although we have obtained our results by perturbing around the CFT or the Gaussian fixed point, the AIII flow equations (2.41b) and (2.42) are in fact exact [92], i.e., valid to all orders in $\lambda$ and $\lambda_{A}$. Clearly, $\lambda>1 / k$ is unphysical, as Eq. (2.42) sends $\lambda_{A}$ (the positive-definite variance of a disorder potential) towards negative values. The case of $\lambda<1 / k$ leads to so-called "Gade" scaling [93], wherein the density of states exhibits a strong (yet integrable) divergence, and the wave functions near zero energy are driven to strong multifractality $[27,29,93]$. This type of behavior occurs for nontopological 2D class AIII systems [27,29,93], which can be localized for sufficiently strong disorder [89]. These include nodal $p$-wave superconductors, wherein $\lambda_{A}$ characterizes quenched orientational fluctuations in the Cooper pair wave function [50]. It is known that the runaway flow $\lambda_{A} \rightarrow \infty$ strongly enhances interaction effects. The result is interactionstabilized Anderson localization $[48,50]$.

One is tempted to conclude that the Wess-ZuminoNovikov-Witten conformal field theories do not describe the 
low-energy physics of classes AIII and DIII (even in the absence of interactions), because the RG flow appears to require fine-tuning to avoid the above-described instabilities. However, there is good evidence to the contrary. First, one can show that the Landauer spin conductance in classes CI and AIII for the disordered Dirac theories in Eqs. (2.1) and (2.13) is universal, independent of the disorder strength [19,30,64]. For a class CI or AIII TSC with winding number $v$, one has

$$
\sigma_{x x}^{s}=\frac{|v|}{\pi h}\left(\frac{\hbar}{2}\right)^{2} .
$$

[This is the analog of the Landauer conductance for $|\nu|$ species of massless Dirac electrons doped to the Dirac point. In Eq. (2.43), $\hbar / 2$ replaces the electric charge $e$.] For class DIII, one can artificially double the theory to introduce a fictitious U(1) charge [80]. Then the arguments in Ref. [64] imply that the associated charge conductance is universal, and the thermal conductivity obtains via the Wiedemann-Franz relation. The DIII Majorana system carries half the value obtained in the doubled system [80], leading to the universal result

$$
\kappa=\frac{k \pi^{2}}{6} \frac{k_{B}^{2}}{\pi h} T
$$

for $k=|v|$ valleys ( $v$ denotes the bulk winding number). Here, we ignore the effects of irrelevant perturbations such as the random modulation of the Fermi velocity [18].

The Landauer conductance is a universal coefficient times the parameter $k$ (proportional to the number of valleys). Except for the Wess-Zumino-Novikov-Witten term, Eq. (2.38) has the same structure as the replicated nonlinear sigma model for quantum diffusion in the appropriate symmetry class, which can arise from any 2D microscopic theory with the appropriate combination of time-reversal, particle-hole, and chiral symmetries $[2,23]$. Then the coupling $\lambda$ is proportional to the dimensionless resistance, and should be pegged to the universal value $1 / k$.

Further evidence that $\lambda=1 / k$ is protected comes from considering interaction corrections to the conductance. We have found that the Altshuler-Aronov corrections to $\lambda$ vanish for $\lambda=1 / k$ in all three classes, as discussed in Ref. [51] and reviewed in Sec. II D, below. This result is valid to all orders in one of the interactions (for classes CI and AIII), but is perturbative in $\lambda$. Second, we have also extended the argument of Ref. [64] to the Hartree and Fock spin conductance corrections in the Dirac language for classes CI and AIII. We find that these vanish exactly [51] in every realization of the disorder. The consistency of these two different approaches requires that $\lambda=1 / k$ in classes $\mathrm{CI}$ and AIII.

Finally, in a separate work [61], we have computed the density of states exponent $x_{1} / z$ [Eq. (2.28)] and the multifractal spectrum $\tau(q)$ [Eq. (2.35)] numerically for a class AIII TSC Dirac Hamiltonian with two valleys $(k=2)$. Our results [61] are consistent with the CFT, Eqs. (2.30) and (2.36).

We therefore expect that the strict conformal invariance of the noninteracting TSC surface states is topologically protected. This implies that a disordered, noninteracting class AIII surface will not exhibit Gade scaling [27,29,93]. We expect that the thermal metal phase described in Ref. [80] is not realized at the surface of a class DIII TSC.

\section{Interactions: methods, multifractality, and Chalker scaling}

In what follows, we discuss the effects of interactions on disordered TSC surface states from multiple perspectives. First, we enumerate all short-ranged four-fermion interactions consistent with bulk symmetries (e.g., time-reversal invariance). We do not consider long-ranged Coulomb interactions, since these should be screened by the bulk superfluid. Using the conformal field theories (CFTs) described in Sec. II B, we have computed the exact scaling dimensions of each to determine their relevance or irrelevance as perturbations to the noninteracting fixed points. We report these results and discuss their implications. We also give the full one-loop RG equations for the Wess-Zumino-Novikov-Witten Finkel'stein nonlinear sigma models (WZNW-FNLsMs) in classes CI and AIII. These are effective field theories obtained from non-Abelian bosonization of the associated CFTs. The one-loop RG is useful in the $k \gg 1$ large winding number/many-valley limit. These equations can include the effects of interactions to all orders, but are valid only to the lowest order in $1 / k$. We discuss the stability of TSC surface states by combining results and insights gained from the CFT and WZNW-FNLsM calculations.

The multifractal [21] character of critically delocalized wave functions [22,23] plays a crucial role in modulating interaction effects for TSC surface states. This is because generic short-ranged interactions are strongly irrelevant in the absence of disorder. Multifractality means that individual wave functions are highly inhomogeneous in space, with large accumulations in rare regions [22,23]. Yet the remarkable phenomenon of Chalker scaling [37-39] implies that such accumulations in single particle wave functions with nearby energy eigenvalues are strongly correlated. In other words, wave functions close in energy exhibit overlapping peaks in position space. This can be understood by defining the energy-split inverse participation ratio [39,61] [cf. Eq. (2.32)]

$$
\mathcal{P}_{2}(\varepsilon, L) \equiv \int d^{2} \mathbf{r}\left|\psi_{0}(\mathbf{r})\right|^{2}\left|\psi_{\varepsilon}(\mathbf{r})\right|^{2},
$$

where $\psi_{\varepsilon}(\mathbf{r})$ is a representative wave function with eigenenergy $\varepsilon$. For critically delocalized states such as those near zero energy at the surface of a dirty topological superconductor, one has the asymptotic behaviors

$$
\begin{aligned}
P_{2}(\varepsilon \rightarrow 0, L) & \sim \frac{1}{L^{\tau(2)}}, \\
P_{2}(\varepsilon, L \rightarrow \infty) & \sim \frac{\varepsilon^{-\mu}}{L^{2}}, \quad \mu=\frac{2-\tau(2)}{z} \geqslant 0 .
\end{aligned}
$$

Here, $\tau(2)$ is the second multifractal exponent [Eqs. (2.33), (2.35), and (2.36)], and $z$ is the dynamic critical exponent [Eqs. (2.28)-(2.30)]. The first limit in Eq. (2.46a) recovers the second multifractal moment of a given wave function, which defines the exponent $\tau(2)$. A nonzero value for this exponent implies that the state is delocalized. The opposite limit in Eq. (2.46b) is (generalized) [61] Chalker scaling, and shows that different wave functions maintain mutual power-law correlations with respect to the energy separation. We have verified this result numerically for the single valley Dirac fermion surface state of a class AIII TSC in Ref. [61]. Since multifractal wave functions with nearby 
energies exhibit a high degree of correlation, the effects of short-ranged interactions can be amplified (suppressed) in peaks (valleys) of the probability densities associated to these states $[15,40,41,76]$.

The situation is completely different in an Anderson insulator. In the latter, two distinct wave functions $\psi_{\varepsilon}(\mathbf{r})$ and $\psi_{\varepsilon^{\prime}}(\mathbf{r})$ close in energy have negligible overlap in their position space probability densities, so that

$$
\left|\psi_{\varepsilon}(\mathbf{r})\right|^{2}\left|\psi_{\varepsilon^{\prime}}(\mathbf{r})\right|^{2} \sim 0 \forall \mathbf{r} .
$$

This result assumes that $\left|\varepsilon-\varepsilon^{\prime}\right|$ is much smaller than the level spacing inside a characteristic localization volume. As a result, weak short-ranged interactions have negligible effect in the insulating phase (at zero temperature). See also Fig. 1.

Given the above, multifractal scaling can enhance the matrix elements of interactions $[15,40,41,76]$. For a coupling strength $U$ associated to a particular four-fermion interaction, this is encoded in the "tree level" RG equation [15]

$$
\frac{d \ln U}{d l}=x_{1}-x_{2}^{(U)}+\boldsymbol{O}(U),
$$

where $x_{1}$ is the scaling dimension of the disorder-averaged density of states [cf. Eq. (2.28)], while $x_{2}^{(U)}$ is the scaling dimension of the four-fermion interaction operator. $\{$ These dimensions are computed in the zero energy, $(2+0)$-D theory; the link to the low-energy sector of the $(2+1)$-D theory is assured for (nearly) extended states by Chalker scaling [37-39,61].\} Equation (2.47) is derived in Appendix C.

In the clean limit $x_{2}^{(U)}=2 x_{1}$, independent of the details of the interaction. Then $U$ is relevant (irrelevant) for $x_{1}<0$ $\left(x_{1}>0\right)$, corresponding to a diverging (vanishing) density of states at zero energy [Eq. (2.28)]. By contrast, for multifractal states in a disordered system, the exponent $x_{2}^{(U)}$ satisfies the bound $x_{2}^{(U)} \geqslant x_{2}$. Here, $x_{2}$ denotes the scaling dimension for the (disorder-averaged) second moment of the local density of states. This is related to the second multifractal exponent via $\tau(2)=2\left(1-x_{1}\right)+x_{2}$ [Eq. (2.34)]. A fourfermion interaction $U$ that saturates the bound $x_{2}^{(U)}=x_{2}$ is maximally relevant [77]; whether this occurs for a particular interaction channel depends upon the symmetry structure (in valley and spin space) of its matrix elements. A key difference from the clean case is that $x_{2}<2 x_{1}$, because the $x_{q}$ characterize moments of a probability distribution [94]. This is the mechanism by which multifractality can enhance interaction effects.

\section{Surface state stability and phase diagrams}

In Sec. II D 1, we review and elaborate upon previous results [15] for class CI. New results for AIII and DIII appear in II D 2 and II D 3. These are derived in Sec. III.

\section{Spin $S U(2)$ symmetry: Class $C I$}

Consider a class CI TSC with winding number $v= \pm 2 k$. The noninteracting class CI surface theory is defined by the Hamiltonian in Eq. (2.1), with the conformal decomposition in Eq. (2.7). We assume that interparticle interactions preserve the bulk spin SU(2) and time-reversal symmetries. To simplify the presentation, we will also assume invariance of the interactions under valley $\mathrm{Sp}(2 k)$ transformations, although this is not necessary.

The structure of four-fermion interaction operators can be obtained two ways. A formal method is to enumerate holomorphic fermion bilinears that transform in different irreducible representations of the spin and valley symmetry groups. The symmetry-allowed four-fermion terms are invariant "diagonal" products of holomorphic and antiholomorphic bilinears. Terms constructed in this way are automatic eigenoperators of the conformal group, and their scaling dimensions are easily obtained. This is the method we employ in Sec. III.

Alternatively, one can construct interactions from products of "physical" operators such as currents, densities, etc. The form of the interactions can be largely inferred from principles of disorder-dominated quantum hydrodynamics [15,46-50]. Because spin is fully conserved, we expect a spin exchange ("triplet") interaction of the form $\vec{S} \cdot \vec{S}$ will play an important role, where $\vec{S}(\mathbf{r})$ is the spin density, e.g., $S^{z}=\psi^{\dagger} \psi / 2$ [Eq. (2.5)]. We can also have a spin current-current interaction of the form $\vec{J}_{S} \cdot \vec{J}_{S}$. Finally, time-reversal symmetry implies that a BCS pairing interaction could induce pair formation. As discussed in Sec. II A 4, the Dirac mass operator $m(\mathbf{r})=$ $\psi^{\dagger} \hat{\sigma}^{3} \psi$ is spin $\mathrm{SU}(2)$ symmetric, but breaks time-reversal symmetry. A nonzero $\langle m\rangle$ opens a gap. In a microscopic lattice model [4], it can be shown [15] that $m(\mathbf{r})$ is an imaginary spin-singlet (e.g., $s$-wave) pairing amplitude, i.e.,

$$
m(\mathbf{r}) \sim-i c_{\uparrow}^{\dagger}(\mathbf{r}) c_{\downarrow}^{\dagger}(\mathbf{r})+i c_{\downarrow}(\mathbf{r}) c_{\uparrow}(\mathbf{r}),
$$

where $c_{\sigma}(\mathbf{r})$ annihilates a lattice electron. We can therefore write an attractive BCS interaction as $-m^{2}(\mathbf{r})$. The formal and intuitive approaches to interaction operator construction are connected through the Fierz identities in Table I.

The interaction Hamiltonian is [15]

$$
\begin{aligned}
H_{\mathrm{CI}}^{(I)}= & \int d^{2} \mathbf{r}\left[U\left(m_{a} m_{a}-4 \vec{S}_{a} \cdot \vec{S}_{a}\right)+V J_{S a}^{\gamma} \bar{J}_{S a}^{\gamma}\right. \\
& \left.+W\left(3 m_{a} m_{a}+4 \vec{S}_{a} \cdot \vec{S}_{a}-\frac{1}{k} J_{S a}^{\gamma} \bar{J}_{S a}^{\gamma}\right)\right] .
\end{aligned}
$$

Here, we have included the replica label $a$, which is summed. In Eq. (2.48), $J_{S a}^{\gamma}\left(\bar{J}_{S a}^{\gamma}\right)$ denotes the holomorphic (antiholomorphic) $\gamma$-spin current $(\gamma \in\{1,2,3\})$. The coupling constants $U, V, W$ are assigned to particular combinations of fourfermion terms. These combinations are eigenoperators [15] of the conformal group with well-defined scaling dimensions at the disordered, noninteracting fixed point. We note that the minimal case $k=1$ (two valleys) is special, in that the $W$-channel interaction does not exist. For that case only, $J_{S a}^{\gamma} \bar{J}_{S a}^{\gamma}=3 m_{a} m_{a}+4 \vec{S}_{a} \cdot \vec{S}_{a}$.

Using the $\operatorname{Sp}(2 n)_{k}$ CFT, one can compute the scaling dimensions $\left\{x_{2}^{(U, V, W)}\right\}$. Combining each of these with $x_{1}$ [Eqs. (2.29) and (2.30)] in Eq. (2.47), one finds [15]

$$
\begin{aligned}
\frac{d U}{d l} & =\frac{1}{2(k+1)} U+\boldsymbol{O}\left(A^{2}, A B\right), \\
\frac{d V}{d l} & =-\frac{(4 k+3)}{2(k+1)} V+\boldsymbol{O}\left(A^{2}, A B\right), \\
\frac{d W}{d l} & =-\frac{3}{2(k+1)} W+\boldsymbol{O}\left(A^{2}, A B\right),
\end{aligned}
$$


where $A, B \in\{U, V, W\}$. These equations imply that $U$ is relevant for any $k$, while $V$ and $W$ are always irrelevant.

In the clean limit, all short-ranged interactions are equally irrelevant, so that (for example)

$$
\frac{d U}{d l}=-U+\boldsymbol{O}\left(A^{2}, A B\right) .
$$

Equation (2.49a) implies that disorder promotes this interaction to a relevant perturbation to the noninteracting TSC surface theory. This can be understood as a strong multifractal enhancement of the matrix elements in the $U$-interaction channel, as discussed above in Sec. II C.

To be precise, the enhancement of $U$ is due to the fact that $x_{2}^{(U)}=x_{2}$, i.e., that the dimension of the interaction operator is the same as that of the second LDoS moment (averaged over disorder realizations). The latter is related to the multifractal dimension $\tau(2)$ [Eq. (2.34)]. (See also the discussion in Sec. II C, above.) For a dirty class CI surface, the conformally invariant fixed point has $x_{2}=0$ for all $k$, while $x_{1}=1 / 2(k+1)$ [85]. By contrast, the other interactions have $x_{2}^{(V, W)}>x_{2}$ such that these remain irrelevant as in the clean limit. [In the clean limit, $x_{2}=2 x_{1}=2$, so that Eq. (2.47) reduces to Eq. (2.50).]

Because $U$ couples to the difference of repulsive (positivedefinite) spin-spin $\vec{S} \cdot \vec{S}$ and cooper pairing $m^{2}$ operators, we interpret the RG flow away from the noninteracting CFT to imply that arbitrarily weak interactions break time-reversal symmetry spontaneously. The initial sign of $U$ depends upon microscopic details, but a flow $U \rightarrow+\infty$ signals a magnetic instability in which we expect $\langle\vec{S}\rangle \neq 0$, at least in local regions. A flow $U \rightarrow-\infty$ signals an instability towards imaginary pairing of surface state quasiparticles, leading to a nonzero Dirac mass $\langle m\rangle \neq 0$ and the surface spin quantum Hall effect, as discussed above in Sec. II A 4. These results for class CI are summarized in Fig. 2.

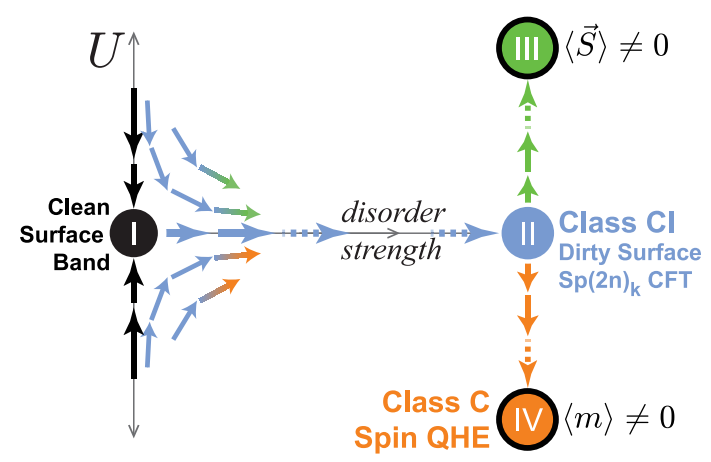

FIG. 2. (Color online) Phase portrait sketch for the surface physics of a $3 \mathrm{D}$ class $\mathrm{CI}$ topological superconductor. The vertical axis is the interaction strength $U$ [Eq. (2.48)], while the horizontal axis measures nonmagnetic disorder. Although the noninteracting system has a disorder-stabilized phase with delocalized ("protected") surface states (II), it is destroyed by arbitrarily weak interactions [Eq. (2.49a)]. Instead, at zero temperature, we expect that the surface exhibits broken spin symmetry $[U>0 \Rightarrow$ (III)], or the spin quantum Hall effect $[U<0 \Rightarrow(\mathbf{I V})]$. In either scenario, interactions break time-reversal symmetry spontaneously.
The dimensions in Eq. (2.49) are exact, and were obtained using the representation theory of the $\operatorname{Sp}(2 n)_{k}$ affine Lie algebra. A drawback of this approach is that it is very difficult to compute higher order ("loop") corrections to these equations in the interaction strengths, or to determine the backreaction of interactions upon the spin or thermal conductance. The problem is that these corrections entail virtual frequency integrations, i.e., the evaluation of correlation functions at one or more nonzero frequencies. Formally, these frequencies are relevant perturbations to the $(2+0)$-D CFT. In principle, one could attempt a version of RG-improved conformal perturbation theory to construct these objects, but such a project is likely to run aground due to infrared divergences.

Fortunately, there is an easier way forward, at least in the limit of large topological winding numbers $|\nu|=2 k$. For $k \gg$ 1, the non-Abelian bosonization of this CFT [Eq. (2.38) with $\lambda=1 / k$ ] becomes a weakly-coupled nonlinear sigma model, which is amenable to a perturbative $\mathrm{RG}$ approach (with $1 / k$ as the small parameter). We can generalize this to a $(2+1)-\mathrm{D}$ imaginary time version that allows direct incorporation of the interactions. The result is a class CI Finkel'stein nonlinear sigma model [46-49] with a Wess-Zumino-Novikov-Witten term (WZNW-FNLsM). The action for this theory is

$$
\begin{aligned}
S_{\mathrm{CI}}= & \frac{1}{\lambda} \int \frac{d^{2} \mathbf{r}}{8 \pi} \operatorname{Tr}\left[\nabla \hat{Q}^{\dagger} \cdot \nabla \hat{Q}\right]+k S_{\mathrm{WZNW}} \\
& -\eta \int d^{2} \mathbf{r} \operatorname{Tr}\left[\hat{\omega}_{N}\left(\hat{Q}+\hat{Q}^{\dagger}\right)\right] \\
& -\int d \tau d^{2} \mathbf{r}\left(4 \Gamma_{t} \overrightarrow{\mathcal{S}}_{a} \cdot \overrightarrow{\mathcal{S}}_{a}+\Gamma_{c} \mathcal{M}_{a} \mathcal{M}_{a}\right),
\end{aligned}
$$

where $S_{\text {WZNW }}$ is the Wess-Zumino-Novikov-Witten term [Eq. (2.39)]. Here, $\hat{Q}(\mathbf{r}) \rightarrow Q_{a, b}^{s, s^{\prime}}\left(\omega_{n}, \omega_{n}^{\prime} ; \mathbf{r}\right)$ is a dynamical matrix field with indices in replica $(a, b)$, spin $\left(s, s^{\prime}\right)$, and Matsubara frequency $\left(\omega_{n}, \omega_{n}^{\prime}\right)$ spaces. Formally, $\hat{Q}$ is a group element in $\operatorname{Sp}(2 n N)$, where $n(N)$ denotes the number of replicas (Matsubara frequencies), and we are to take $n \rightarrow 0$, $N \rightarrow \infty$ such that $n N \rightarrow 0$. The first three terms in Eq. (2.51) define the noninteracting theory. In the third term, $\hat{\omega}_{N}$ is a diagonal matrix of Matsubara frequencies. This term does not appear in the $(2+0)$-D Wess-Zumino-Novikov-Witten CFT [Eq. (2.38)], since the latter describes the properties at $\omega_{N}=0$.

Four-fermion interactions are encoded in the final term of Eq. (2.51). Interactions involve bilinears of $Q_{a, b}^{s, s^{\prime}}\left(\tau, \tau^{\prime}\right)$ that are local (diagonal) in replica and imaginary time indices $[46,47]$. The operators

$$
\begin{aligned}
\overrightarrow{\mathcal{S}}_{a}(\tau, \mathbf{r}) & \equiv \frac{1}{2} \operatorname{Tr}_{s}\left\{\hat{\vec{s}}\left[\hat{Q}_{a a}(\tau, \tau ; \mathbf{r})+\hat{Q}_{a a}^{\dagger}(\tau, \tau ; \mathbf{r})\right]\right\}, \\
\mathcal{M}_{a}(\tau, \mathbf{r}) & \equiv \operatorname{Tr}_{s}\left[\hat{Q}_{a a}(\tau, \tau ; \mathbf{r})-\hat{Q}_{a a}^{\dagger}(\tau, \tau ; \mathbf{r})\right]
\end{aligned}
$$

are the sigma model versions of the spin and BCS mass densities, respectively. In these equations, the trace $\operatorname{Tr}_{s}$ runs over spin components; $\hat{\vec{s}}$ is the vector of Pauli matrices acting on spin space. The interaction operators conjugate to the coupling strengths $\Gamma_{t}$ and $\Gamma_{c}$ in Eq. (2.51) correspond to the spin exchange $\vec{S} \cdot \vec{S}$ and cooper pairing $m^{2}$ operators in Eq. (2.48). We could also include the spin current-current interaction, but this channel becomes strongly irrelevant in the $k \gg 1$ limit [Eq. (2.49b)]. 
By parameterizing $\hat{Q}$ in terms of some unconstrained coordinates, it is straightforward to implement a perturbative RG scheme using Eq. (2.51). The nontopological version (lacking the Wess-Zumino-Novikov-Witten term) was studied in Ref. [49]. At one-loop, the WZNW term modifies only the weak localization [95] and Altshuler-Aronov corrections [96] to the inverse spin conductance [51]. The one-loop RG equations are given by $[49,51]$

$$
\begin{aligned}
\frac{d \lambda}{d l}= & \lambda^{2}\left[1-(k \lambda)^{2}\right] \\
& \times\left\{1+3\left[1+\frac{1-\gamma_{t}}{\gamma_{t}} \ln \left(1-\gamma_{t}\right)\right]-\frac{\gamma_{c}}{2}\right\} \\
\frac{d \gamma_{t}}{d l}= & -\frac{\lambda}{2} \gamma_{c}\left(1-\gamma_{t}\right)\left(1-2 \gamma_{t}\right) \\
\frac{d \gamma_{c}}{d l}= & \frac{\lambda}{2}\left\{-3 \gamma_{t}-2 \gamma_{c}+3 \gamma_{c}\left[\ln \left(1-\gamma_{t}\right)+\gamma_{t}\right]\right\}-\gamma_{c}^{2}
\end{aligned}
$$

These equations are expressed in terms of the relative interaction strengths

$$
\gamma_{t, c} \equiv \frac{4}{\pi \eta} \Gamma_{t, c}
$$

where $\eta$ is the coefficient of the frequency term in Eq. (2.51). Equation (2.53) incorporates corrections to second homogeneous order [97] in $\lambda$ and $\gamma_{c}$, but includes corrections to all orders in $\gamma_{t}$.

Consider first Eq. (2.53a). In Sec. II B 2, we argued that the parameter $\lambda$ is proportional to the inverse dc spin conductance. Then the first term (" 1 ") on the second line is a weak localization correction [95]. The second and third terms are Altshuler-Aronov conductance corrections $[49,96]$ due to the spin triplet $\gamma_{t}$ and Cooper channel $\gamma_{c}$ interactions, respectively. At the conformal fixed point $\lambda=1 / k$, all corrections are suppressed in Eq. (2.53a) [51].

For noninteracting quasiparticles on the surface of a disordered class CI TSC, it is known $[30,64]$ that the Landauer spin conductance is unmodified from its clean value in Eq. (2.43). Equation (2.53a) implies that interaction corrections are also suppressed. This statement is valid to lowest order in $\lambda=1 / k$.

In Ref. [51], we consider the lowest order Hartree and Fock interaction corrections to the spin conductance in the disordered Dirac description of the TSC surface. Without resorting to the CFT, we demonstrate that these vanish exactly. This is consistent with Eq. (2.53a) only for $\lambda=1 / k$. We therefore argue that the Landauer spin conductance is universal and given by Eq. (2.43), so long as time-reversal symmetry is not broken (spontaneously or by external means). This implies that topological superconductor surface states are similar to the chiral edge modes of the quantum Hall effect, in that the bulk topological winding number is encoded in a universal transport coefficient.

In the remainder, we will set $\lambda=1 / k$ and assume that higher order corrections do not modify this. We turn to the interaction flow equations (2.53b) and (2.53c). To compare to the CFT results, we define

$$
\gamma_{U} \equiv \frac{1}{4}\left(\gamma_{c}-3 \gamma_{t}\right), \gamma_{W} \equiv \frac{1}{4}\left(\gamma_{c}+\gamma_{t}\right) .
$$

The interaction part of Eq. (2.51) is proportional to

$$
\begin{aligned}
& -\int d \tau d^{2} \mathbf{r}\left[\gamma_{U}\left(\mathcal{M}_{a} \mathcal{M}_{a}-4 \overrightarrow{\mathcal{S}}_{a} \cdot \overrightarrow{\mathcal{S}}_{a}\right)\right. \\
& \left.\quad+\gamma_{W}\left(3 \mathcal{M}_{a} \mathcal{M}_{a}+4 \overrightarrow{\mathcal{S}}_{a} \cdot \overrightarrow{\mathcal{S}}_{a}\right)\right],
\end{aligned}
$$

which has the same structure as Eq. (2.48) with the identification $\gamma_{U, W} \Leftrightarrow U, W$. Linearizing Eqs. (2.53b) and (2.53c) in the interaction strengths gives [15]

$$
\begin{aligned}
\frac{d \gamma_{U}}{d l} & =\frac{1}{2 k} \gamma_{U}, \\
\frac{d \gamma_{W}}{d l} & =-\frac{3}{2 k} \gamma_{W} .
\end{aligned}
$$

These agree with Eq. (2.49) to lowest order in $1 / k$ for the corresponding coupling strengths.

The one-loop Eqs. (2.53b) and (2.53c) possess only the noninteracting fixed point $\gamma_{U}=\gamma_{W}=0$, which as we have seen is unstable. The full nonlinear structure of these equations is somewhat complicated. However, the main physics is captured by retaining only the $-\gamma_{c}^{2}$ term in Eq. (2.53c), and linearizing the rest. This term represents the BCS instability towards imaginary Cooper pairing of the surface quasiparticles. Its appearance in the disordered theory is a manifestation of Anderson's theorem $[49,98]$ (the interaction operator $\mathcal{M}_{a} \mathcal{M}_{a}$ is time-reversal even, although $\mathcal{M}_{a}$ is odd). In Fig. 3, we plot the RG flows in the $\gamma_{U}-\gamma_{W}$ plane for the full flow Eqs. (2.53b) and $(2.53 \mathrm{c})$ (blue) and the simplified equations (red) defined via

$$
\begin{aligned}
\frac{d \gamma_{U}}{d l} & =\frac{1}{2 k} \gamma_{U}-\frac{1}{4}\left(\gamma_{U}+3 \gamma_{W}\right)^{2}, \\
\frac{d \gamma_{W}}{d l} & =-\frac{3}{2 k} \gamma_{W}-\frac{1}{4}\left(\gamma_{U}+3 \gamma_{W}\right)^{2} .
\end{aligned}
$$

Equation (2.58) is obtained by adding the BCS interaction term $-\gamma_{c}^{2}$ to Eq. (2.57). Figure 3 illustrates that the dominant interaction instability is towards the direction of BCS pairing. In accordance with our schematic phase diagram in Fig. 2, we expect that a flow $\gamma_{c} \rightarrow-\infty$ (i.e., $\gamma_{U, W} \rightarrow-\infty$ ) signals the development of the surface spin quantum Hall effect, with $\left\langle\mathcal{M}_{a}\right\rangle \sim\left\langle m_{a}\right\rangle \neq 0$. (See the discussion in Sec. II A 4.)

\section{Spin U(1) symmetry: Class AIII}

For a TSC in class AIII, only a U(1) remnant of spin SU(2) symmetry is preserved. We associate this with rotations about the $z$ axis in spin space. The interaction Hamiltonian is

$$
\begin{aligned}
H_{\mathrm{AIII}}^{(I)}= & \int d^{2} \mathbf{r}\left[\frac{U}{2}\left(m_{a} m_{a}-4 S_{a}^{z} S_{a}^{z}-\frac{4}{k} J_{a} \bar{J}_{a}\right)\right. \\
& \left.+V J_{a} \bar{J}_{a}+\frac{W}{2}\left(m_{a} m_{a}+4 S_{a}^{z} S_{a}^{z}\right)\right] .
\end{aligned}
$$

The structure of Eq. (2.59) is similar to the CI case Eq. (2.48), except that only the $z$ components of the spin density and current appear $\left(J_{a} \equiv J_{S a}^{z}\right.$ denotes the $z$-spin current). 

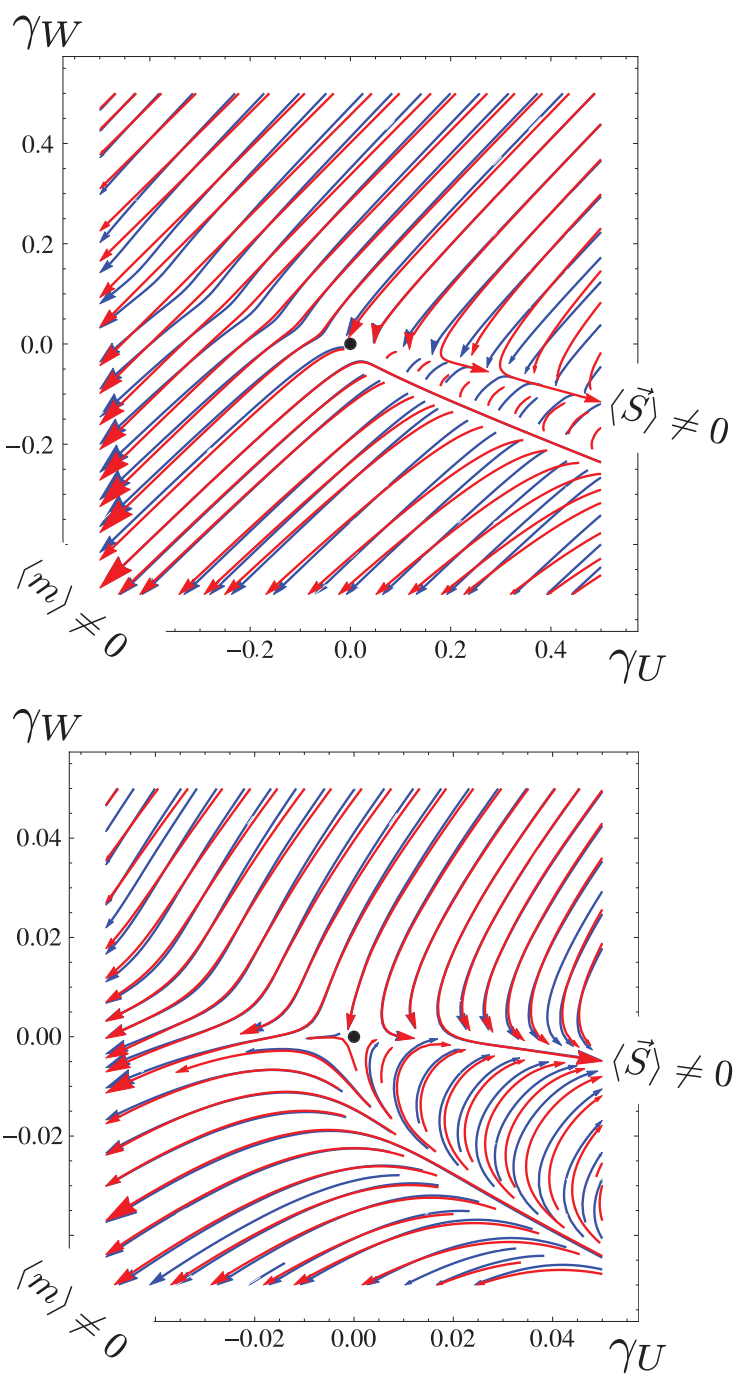

FIG. 3. (Color online) Class CI RG flows in the interaction coupling space $\gamma_{U}, \gamma_{W}$. The interaction $\gamma_{U}\left(\gamma_{W}\right)$ is a relevant (irrelevant) perturbation to the noninteracting fixed point (marked by the black dot), in either the CFT [Eqs. (2.48) and (2.49)] or WZNW-FNLsM languages [Eqs. (2.56) and (2.57)]. The flow in blue is the full one-loop WZNW-FNLsM result [Eqs. (2.53b) and (2.53c)], while that in red is the simplified flow in Eq. (2.58) that neglects all nonlinearities except the $-\gamma_{c}^{2}$ BCS term in Eq. (2.53c). In these plots, we have set $k=1 / \lambda=30$. The bottom panel is a zoom of the top in the vicinity of the noninteracting fixed point. The flows are consistent with the schematic phase diagram in Fig. 2, and further suggest that BCS pairing $\left(\gamma_{U}, \gamma_{W} \rightarrow-\infty\right)$ is the dominant instability.

Alternatively, we can write

$$
\begin{aligned}
H_{\mathrm{AIII}}^{(I)} & =\int d^{2} \mathbf{r}\left[U_{c} m_{a} m_{a}+U_{t} 4 S_{a}^{z} S_{a}^{z}+U_{j} J_{a} \bar{J}_{a}\right], \\
U_{t, c} & =\frac{1}{2}(W \mp U), \quad U_{j}=V-\frac{2}{k} U .
\end{aligned}
$$

Evaluating Eq. (2.47) for each of the interaction channels in Eq. (2.59) using the $\mathrm{U}(n)_{k}$ CFT, we obtain

$$
\frac{d U}{d l}=\left(\frac{1}{k^{2}}-\lambda_{A}\right) U+\boldsymbol{O}\left(A^{2}, A B\right),
$$

$$
\begin{aligned}
& \frac{d V}{d l}=\left(\frac{1-2 k^{2}}{k^{2}}-\lambda_{A}\right) V+\boldsymbol{O}\left(A^{2}, A B\right), \\
& \frac{d W}{d l}=\left(-\frac{3+2 k}{k^{2}}+3 \lambda_{A}\right) W+\boldsymbol{O}\left(A^{2}, A B\right),
\end{aligned}
$$

where $A, B \in\{U, V, W\}$. Equation (2.61) is derived in Sec. III A 2.

First, we note some consistency checks. Equations (2.49) with $k=1$ and (2.61) with $k=2$ (two valleys in each case) are identical for $\lambda_{A}=0$, if we remember that $W$ does not exist for class CI when $k=1$. Instead, $V$ in CI maps to $W$ and $V$ in AIII, which are degenerate in this case. In the absence of the Abelian spin $\mathrm{U}(1)$ vector potential disorder, the two valley class $\mathrm{CI}$ and AIII models are the same because the valley disorder $\mathrm{Sp}(2)=$ $\mathrm{SU}(2)$. Second, Eq. (2.61b) is consistent for $k=1$, wherein the Abelian spin $U(1)$ vector potential disorder $\lambda_{A}$ is a purely marginal perturbation to the clean limit [19]. For that single valley case, only the $V$-interaction channel exists. Because this is a Kac-Moody current-current perturbation $x_{2}^{(V)}=2$, while the scaling dimension of the LDoS is given by $x_{1}=1-\lambda_{A}$ for $k=1$ [19].

Now we examine the criteria for surface state stability. The spin current-current interaction $V$ is always strongly irrelevant. By contrast, $U$ and $W$ may be relevant or irrelevant, depending upon the strength $\lambda_{A}$ of spin current disorder. In particular for $\lambda_{A}<1 / k^{2}$, the $U$-interaction channel is relevant. This is similar to CI, as the associated combination of four-fermion terms involves a difference of repulsive spin triplet and Cooper pairing interactions, Eq. (2.59). By contrast, $W$ becomes relevant for $\lambda_{A}>(3+2 k) / 3 k^{2}$. In this case, the instability of the noninteracting fixed point is mediated by $\lambda_{A}$, similar to the enhancement of interactions in 2D nontopological class AIII systems [48,50]. The amplification of $W$ for sufficiently strong $\lambda_{A}$ is a multifractal enhancement, but now due to the spin U(1) current disorder.

We conclude that class AIII exhibits a disorder-dependent window of stability wherein all three interactions $U, V, W$ are irrelevant,

$$
\frac{1}{k^{2}}<\lambda_{A}<\frac{3+2 k}{3 k^{2}} .
$$

This region is plotted in Fig. 4.

What happens for $\lambda_{A}$ outside of Eq. (2.62)? Within the CFT approach, we can only say that the noninteracting fixed point is unstable. As in class CI, in the large $k \gg 1$ limit, the non-Abelian bosonization of the $\mathrm{U}(n)_{k}$ CFT becomes a weakly coupled sigma model, with $1 / k$ as a small parameter. Writing the $(2+1)$-D imaginary time version and incorporating interactions, we get the action

$$
\begin{aligned}
S_{\mathrm{AIII}}= & \frac{1}{\lambda} \int \frac{d^{2} \mathbf{r}}{8 \pi} \operatorname{Tr}\left[\nabla \hat{U}^{\dagger} \cdot \nabla \hat{U}\right]+k S_{\mathrm{WZNW}} \\
& -\frac{\lambda_{A}}{\lambda^{2}} \int_{\mathbf{r}} \frac{d^{2} \mathbf{r}}{8 \pi}\left[\operatorname{Tr}\left(\hat{U}^{\dagger} \nabla \hat{U}\right)\right]^{2} \\
& -\eta \int d^{2} \mathbf{r} \operatorname{Tr}\left[\hat{\omega}_{N}\left(\hat{U}+\hat{U}^{\dagger}\right)\right] \\
& -\sum_{a=1}^{n} \int d \tau d^{2} \mathbf{r}\left(4 \Gamma_{t} \mathcal{S}_{a}^{z} \mathcal{S}_{a}^{z}+\Gamma_{c} \mathcal{M}_{a} \mathcal{M}_{a}\right) .
\end{aligned}
$$




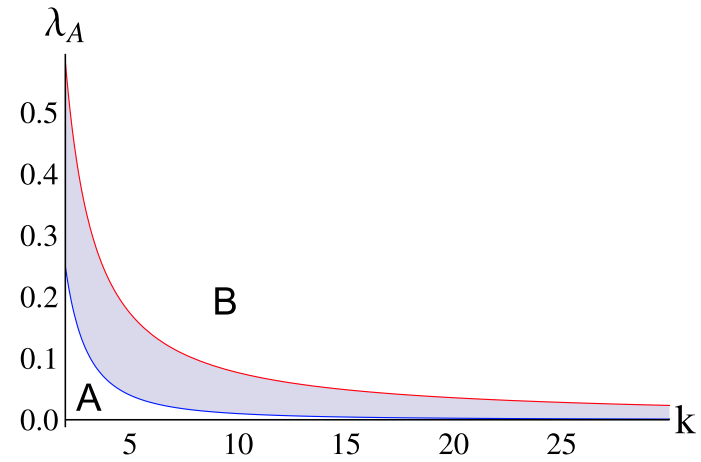

FIG. 4. (Color online) Spin current disorder-stabilized AIII TSC surface states. In the presence of spin U(1) current disorder $\lambda_{A}$, the disordered, noninteracting CFT description of a class AIII TSC with winding number $|v|=k$ can be stable to weak interaction effects. The stable window is the shaded region, given by Eq. (2.62). By contrast, the interaction channel $U(W)$ is relevant in region $\mathrm{A}(\mathrm{B})$ [Eq. (2.61)].

The matrix field $\hat{U} \rightarrow U_{a, b}\left(\omega_{n}, \omega_{n}^{\prime} ; \mathbf{r}\right)$ is a group element of $\mathrm{U}(n N)$, with $n \rightarrow 0$ replicas and $N \rightarrow \infty$ Matsubara frequencies. The interaction are bilinears of the spin and mass operators

$$
\begin{aligned}
\mathcal{S}_{a}^{z}(\tau, \mathbf{r}) & \equiv \frac{1}{2}\left[\hat{U}_{a a}(\tau, \tau ; \mathbf{r})+\hat{U}_{a a}^{\dagger}(\tau, \tau ; \mathbf{r})\right], \\
\mathcal{M}_{a}(\tau, \mathbf{r}) & \equiv \hat{U}_{a a}(\tau, \tau ; \mathbf{r})-\hat{U}_{a a}^{\dagger}(\tau, \tau ; \mathbf{r}) .
\end{aligned}
$$

As in class CI, we exclude the strongly irrelevant currentcurrent interaction.

Relative to the nontopological case [48,50], the WessZumino-Novikov-Witten term modifies only the $\lambda$ and $\lambda_{A}$ RG equations. The one-loop results for the WZNW-FNLsM are $[48,50,51]$

$$
\begin{aligned}
\frac{d \lambda}{d l}= & \lambda^{2}\left[1-(k \lambda)^{2}\right] \mathcal{I}, \\
\frac{d \lambda_{A}}{d l}= & \lambda^{2}\left[1-(k \lambda)^{2}\right]\left(1+\frac{2 \lambda_{A} \mathcal{I}}{\lambda}\right), \\
\frac{d \gamma_{t}}{d l}= & \lambda_{A}\left(1-\gamma_{t}\right)\left(\gamma_{t}+2 \gamma_{c}-2 \gamma_{t} \gamma_{c}\right) \\
& -\lambda\left(1-\gamma_{t}\right)\left(\gamma_{t}+\gamma_{c}-2 \gamma_{t} \gamma_{c}\right), \\
\frac{d \gamma_{c}}{d l}= & \lambda_{A}\left(2 \gamma_{t}+\gamma_{c}\right)-\lambda\left(\gamma_{t}+\gamma_{c}\right) \\
& +\lambda \gamma_{c}\left[2 \ln \left(1-\gamma_{t}\right)+\gamma_{t}\right]-2 \gamma_{c}^{2},
\end{aligned}
$$

where

$$
\mathcal{I} \equiv 2\left[1+\frac{1-\gamma_{t}}{\gamma_{t}} \ln \left(1-\gamma_{t}\right)\right]-\gamma_{c}
$$

is the Altshuler-Aronov [96] spin conductance correction. The relative interactions are

$$
\gamma_{t, c} \equiv \frac{4}{\pi \eta} \Gamma_{t, c}
$$

The noninteracting CFT has $\lambda=1 / k$. Equation (2.65a) and (2.65b) imply that this is a fixed point of $\lambda$ even in the presence of interactions, and that the Abelian disorder parameter $\lambda_{A}$ remains purely marginal in this case. One can say that the topology neutralizes the Altshuler-Aronov correction, and this conclusion holds for CI [Eq. (2.53a)] and DIII as well [Eq. (2.74)] [51].

In Sec. II B 2, we noted that tuning $\lambda<1 / k$ induces runaway flow in $\lambda_{A}$, in the absence of interactions [Eq. (2.42)] [92]. This runaway flow is known as "Gade" scaling $[23,93]$ and is characteristic of nontopological class AIII models in 2D. It is known that Gade scaling induces a density of states divergence at zero energy $[23,92,93]$, as well as strong multifractality in the low-energy wave functions. The runaway $\lambda_{A}$ flow amplifies interactions, so that these are always relevant $[48,50]$.

In Sec. II B 2, we presented evidence that the value of $\lambda$ is pinned to $1 / k$. This includes the universality of the Landauer spin conductance in the absence of interactions, which is proportional to $k$. In the disordered Dirac language of Eq. (2.13), the lowest-order Hartree and Fock interaction corrections to the conductance vanish exactly [51]. In what follows, we set $\lambda=1 / k$ and assume that higher order corrections do not destabilize this. As a result, $\lambda_{A}$ remains a tunable, strictly marginal parameter.

To compare to the CFT, we linearize Eqs. $(2.65 \mathrm{c})$ and (2.65d) and express the results in terms of

$$
\gamma_{U, W} \equiv \gamma_{c} \mp \gamma_{t}
$$

One obtains

$$
\begin{aligned}
\frac{d \gamma_{U}}{d l} & =-\lambda_{A} \gamma_{U}, \\
\frac{d \gamma_{W}}{d l} & =\left(-\frac{2}{k}+3 \lambda_{A}\right) \gamma_{W} .
\end{aligned}
$$

These are consistent with Eq. (2.61) to lowest order in $1 / k$, using the correspondence $\gamma_{U, W} \Leftrightarrow U, W$.

The full one-loop flow Eqs. (2.65c) and (2.65d) possess a nontrivial fixed point that is locally unstable (stable) for $\lambda_{A}<\frac{2}{3 k}\left(\lambda_{A}>\frac{2}{3 k}\right)$. In the latter case, this constitutes a new, interaction-stabilized fixed point. The fixed point coupling strengths $\left\{\gamma_{t}^{*}, \gamma_{c}^{*}\right\}$ are complicated functions of $k$ and $\lambda_{A}$-see Eq. (D1) in Appendix D. The key point is that the interactionstabilized fixed point nucleates from the noninteracting CFT $\left(\left\{\gamma_{t}, \gamma_{c}\right\}=\{0,0\}\right)$ at $\lambda_{A}=2 / 3 k$, and is thus fully controlled for some range of $\lambda_{A}$. As $\lambda_{A}$ is increased above $2 / 3 k$, this new fixed point quickly moves to large $\gamma_{W}$, beyond the regime of reliability for the weak-coupling RG.

We note that Eq. (2.69) seems to imply that $\gamma_{U}$ is irrelevant for any $\lambda_{A}>0$. The CFT result in Eq. (2.61) shows that this is not correct, leading instead to the stability window in Eq. (2.62). [The discrepancy is the $1 / k^{2}$ term in Eq. (2.61a), which would appear in the WZNW-FNLsM treatment only at two loops.] As in class CI, we can get a more intuitive understanding of the interaction plane RG flow by adding only the pure BCS term $-2 \gamma_{c}^{2}=-(1 / 2)\left(\gamma_{U}+\right.$ $\left.\gamma_{W}\right)^{2} \Leftrightarrow-(1 / 2)(U+W)^{2}$ to the CFT results in Eq. (2.61), leading to

$$
\begin{aligned}
\frac{d U}{d l} & =\left(\frac{1}{k^{2}}-\lambda_{A}\right) U-\frac{1}{2}(U+W)^{2}, \\
\frac{d W}{d l} & =\left(-\frac{3+2 k}{k^{2}}+3 \lambda_{A}\right) W-\frac{1}{2}(U+W)^{2} .
\end{aligned}
$$






FIG. 5. (Color online) Simplified RG flow in the $U-W$ interaction plane for class AIII. Flows are plotted for Eq. (2.70). In this figure, $\lambda_{A}=0$, and we have set $k=4$. The red (green) dot denotes the noninteracting (interaction-stabilized) fixed point, which is unstable (stable) for $\lambda_{A}<1 / k^{2}$.

Equation (2.70) neglects most of the nonlinear interaction terms in Eqs. (2.65c) and (2.65d), but gives the same qualitative behavior as these for $\lambda_{A}>1 / k^{2}$. Equation (2.70) possesses a nontrivial fixed point for generic $\lambda_{A}<(k+1) / k^{2}$. The nontrivial fixed point is unstable for $\lambda_{A}$ within the range in Eq. (2.62), wherein the noninteracting CFT $(U=W=0)$ is stable. At the boundaries of this range, the nontrivial fixed point merges with the noninteracting theory. Like the full Eqs. (2.65c) and (2.65d), the nontrivial fixed point is stable for $\lambda_{A}>(3+2 k) / 3 k^{2}$, but quickly moves to strong coupling as $\lambda_{A}$ is increased beyond this threshold value. A new feature of Eq. (2.70) is that the nontrivial fixed point is also stable for $\lambda_{A}<1 / k^{2}$.

In Figs. 5-9, we plot the flow fields in the $U-W$ interaction plane corresponding to Eq. (2.70), for different values of the Abelian disorder strength $\lambda_{A}$. The main takeaway is that for $\lambda_{A}<(1+k) / k^{2}$ there is always a critically delocalized, timereversal invariant fixed point. For $\lambda_{A}$ in the range given by Eq. (2.62), this is the noninteracting $\mathrm{U}(n)_{k}$ CFT. Outside of this range, one finds new interaction-stabilized fixed points. The latter merge with the noninteracting one at the boundaries of Eq. (2.62), Figs. 6 and 8. The trajectory of the nontrivial fixed point as a function of increasing $\lambda_{A}$ is shown in Fig. 10. The flow fields in the $\left\{\gamma_{U}, \gamma_{V}\right\}$ plane for the full one-loop results in Eqs. (2.65c) and (2.65d) are qualitatively the same as those

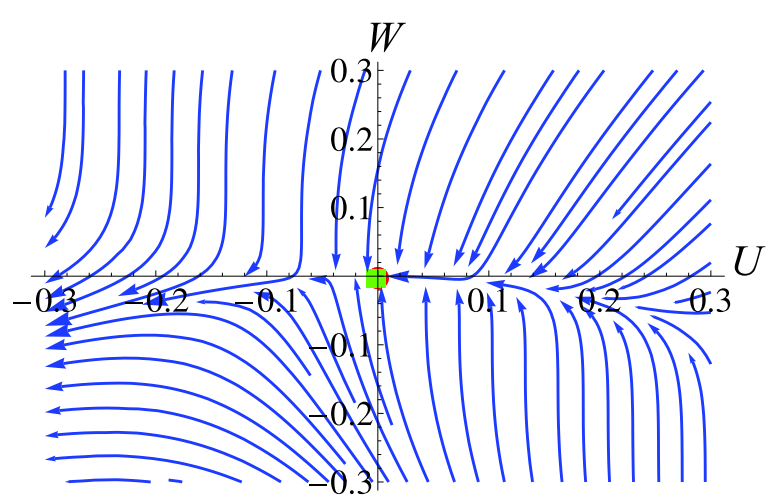

FIG. 6. (Color online) The same as Fig. 5, but for $\lambda_{A}=1 / k^{2}$.

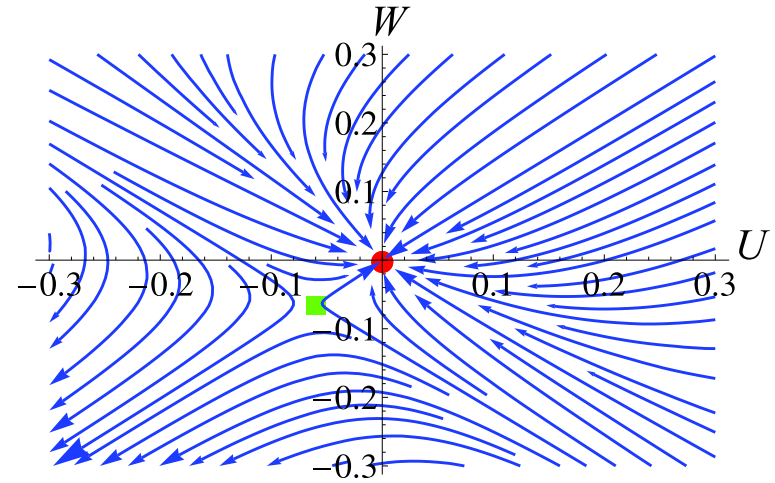

FIG. 7. (Color online) The same as Fig. 5, but for $1 / k^{2}<\lambda_{A}<$ $(3+2 k) / 3 k^{2}$. In this case, the noninteracting fixed point (red) is stable, while the nontrivial fixed point (green) is unstable.

shown in these figures, except that the noninteracting fixed point remains stable for arbitrarily small $\lambda_{A}$ and the nontrivial fixed point merges with this at $\lambda_{A}=0$.

Comparing the interaction flows for class AIII in Figs. 5-9 to class $\mathrm{CI}$ in Fig. 3, it is apparent that the magnetic instability in the latter $(\langle\vec{S}\rangle \neq 0)$ has been replaced by a critically delocalized fixed point. This is either the noninteracting conformal fixed point for $\lambda_{A}$ in the window bounded by Eq. (2.62), or an interaction-stabilized one outside of this. On the other hand, the instability to surface state Cooper pairing $(U, W \rightarrow-\infty)$ is common to both AIII and CI. We conclude that surface states of TSCs in classes AIII and CI are vulnerable to the formation of gapped surface quantum Hall phases with spontaneously broken time-reversal symmetry. As discussed in Sec. II C, the mechanism is the multifractal enhancement of interaction matrix elements, due to the disorder. Unlike class CI, however, class AIII possesses a range of disorder and interaction strengths wherein time-reversal symmetry and critical delocalization are preserved.

\section{No spin symmetry: Class DIII}

Finally we turn to class DIII. Bulk topological superconductors in this class are characterized by the integer-valued winding number $v$. As discussed in the Introduction, the minimal case $k \equiv|v|=1$ corresponding to a single Majorana fermion band is manifestly robust to both disorder and

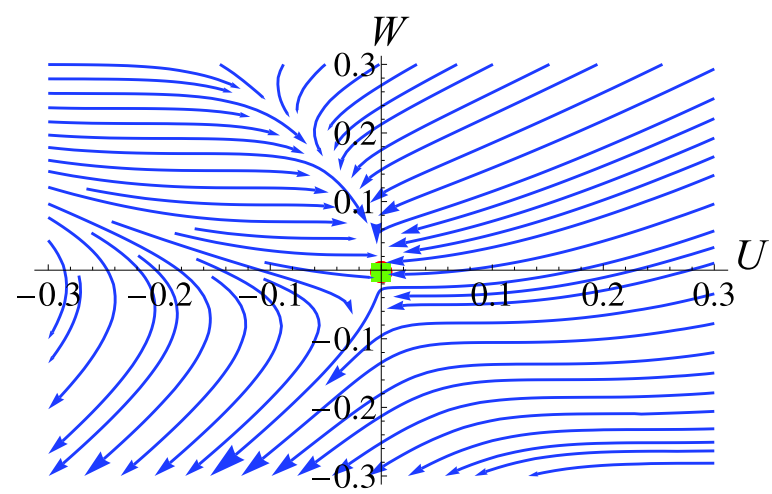

FIG. 8. (Color online) The same as Fig. 5, but for $\lambda_{A}=(3+$ $2 k) / 3 k^{2}$. 


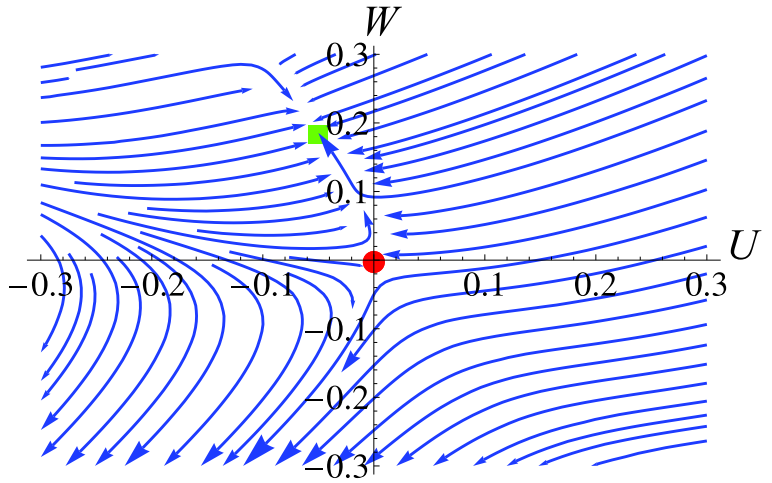

FIG. 9. (Color online) The same as Fig. 5, but for $\lambda_{A}>(3+$ $2 k) / 3 k^{2}$. In this case, the noninteracting fixed point (red) is unstable, while the nontrivial fixed point (green) is stable.

interactions, in the sense that both are strongly irrelevant. The two valley $k=2$ version is equivalent to class AIII with $k=1$, because this theory exhibits an emergent valley $\mathrm{U}(1)$ symmetry.

For $k \geqslant 3$, class DIII admits non-Abelian valley vector potential disorder. Critically delocalized wave functions are described by the $\mathrm{SO}(n)_{k}$ CFT [Eq. (1.1)], with multifractal local density of states (LDoS) fluctuations captured by Eqs. (2.35) and (2.36).

Since spin is not conserved in class DIII, the only valleyinvariant short-ranged interaction is the BCS pairing term [cf. Eqs. (2.48) and (2.59)]. The interaction Hamiltonian is

$$
H_{\mathrm{DIII}}^{(I)}=U \int d^{2} \mathbf{r} m_{a} m_{a},
$$

where $m_{a}=\chi^{\top} \hat{M}_{p} \hat{\sigma}^{3} \chi$ is the Majorana mass operator [Eq. (2.22)]. Using the $\mathrm{SO}(n)_{k}$ CFT, we compute $x_{2}^{(U)}$ and evaluate Eq. (2.47). The result is

$$
\frac{d U}{d l}=-\frac{1}{(k-2)} U+\boldsymbol{O}\left(U^{2}\right) .
$$

Equation (2.72) is derived in Sec. III B 2.

Unlike classes CI and AIII, we conclude that the BCS pairing channel is always irrelevant in class DIII. For sufficiently weak interactions, the surface physics of a disordered DIII TSC with $k \geqslant 3$ is governed by the $\mathrm{SO}(n)_{k}$ CFT, which gives

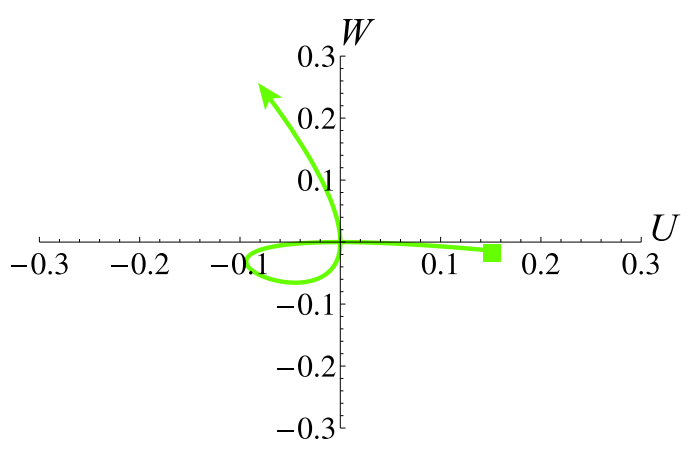

FIG. 10. (Color online) Interaction plane trajectory of the nontrivial fixed point shown in Figs. 5-9 as a function of increasing $\lambda_{A}$. universal predictions for LDoS statistics and which should exhibit the universal thermal conductance given by Eq. (2.44).

Equation (2.72) arises despite the strong disorder enhancement of the average density of states (DoS) $v(\varepsilon)$. Equations (2.28) and (2.30) imply that $\nu(\varepsilon) \sim|\varepsilon|^{-1 /(2 k-3)}$, which diverges as $\varepsilon \rightarrow 0$. By contrast, the average DoS in class CI always vanishes, while it may vanish or diverge in AIII. We would naively expect interactions to play the strongest role in class DIII. This does not occur, however, because the interaction operator $m_{a} m_{a}$ in Eq. (2.71) does not have the same symmetry structure as the second LDoS moment. In class DIII, the $q$ th LDoS moment has the multifractal scaling dimension

$$
x_{q}=-\frac{q^{2}}{(k-2)},
$$

so that $x_{2}=-4 /(k-2)<2 x_{1}=-2 /(k-2)$. [Equation (2.73) is derived in Sec. III B 1.] In evaluating Eq. (2.47), we must determine $x_{2}^{(U)}$, the scaling dimension of the four fermion interaction. In class $\mathrm{CI}$, this is equal to $x_{2}$, implying that the $U$-channel interaction [as defined by Eq. (2.48)] is maximally relevant. The story is different in class DIII, and $x_{2}^{(U)}=0>x_{2}$, as shown in Sec. III B 2.

Physically, this means that while the average density of states is amplified, wave function multifractality does not enhance the matrix elements of the interactions. This is a key concept: it is not enough to say that one has critically delocalized wave functions with multifractal LDoS fluctuations, strongly correlated in energy by Chalker scaling. (See the discussion in Sec. II C.) Rather, one also requires that the particular interaction channel of interest has a nonvanishing overlap with the second LDoS moment, in the sense of common symmetry structures (in spin, valley, and replica indices). In class DIII, the interaction operator has an orthogonal structure to the multifractal moment: the former is symmetric in replica indices, while the latter can be taken to be antisymmetric (Sec. III B 1). A similar disparity between interactions and multifractal moments arises for a 2D unitary metal with short-ranged interactions, which are also irrelevant [47].

One can also formulate a WZNW-FNLsM for class DIII [51]. Because the interaction $U$ is irrelevant, the only new information this provides concerns the beta function for the ratio of the temperature to the thermal conductance [51], proportional to the parameter $\lambda$ which satisfies the one-loop RG equation

$$
\frac{d \lambda}{d l}=\lambda^{2}\left[1-(k \lambda)^{2}\right]\left(-2-\gamma_{c}\right)
$$

As with classes CI and AIII, both the weak antilocalization [80] ("-2") and Altshuler-Aronov (" $-\gamma_{c}$ ") corrections to the conductance are suppressed at the conformal fixed point $\lambda=$ $1 / k$ [51]. Compare to Eqs. (2.53a) (CI), (2.65a), and (2.65b) (AIII).

\section{CONFORMAL ANALYSIS}

In this section, we derive the conformal field theory results discussed in Sec. II. The multifractal spectrum of LDoS fluctuations is computed in Secs. III A 1 and III B 1 for classes AIII and DIII. We enumerate interparticle interaction operators 
for these classes and compute their scaling dimensions in Secs. III A 2 and III B 2. The analysis for class CI has appeared elsewhere [15].

\section{A. Class AIII}

\section{Density of states and multifractal spectrum}

The low-energy effective CFT for a class AIII TSC surface state with $k$ valleys is the $\mathrm{U}(1) \oplus \mathrm{SU}(n)_{k}$ affine Lie algebra [Eq. (1.1)]. Here $n \rightarrow 0$ is the number of replicas. The $(2+0)-$ $\mathrm{D}$ action may be written as [cf. Eq. (2.38)]

$$
\begin{aligned}
S_{\mathrm{AIII}}^{(\mathrm{WZNW})}= & n k\left(1+n k \lambda_{A}\right) \int \frac{d^{2} \mathbf{r}}{8 \pi} \nabla \phi \cdot \nabla \phi \\
& +k \int \frac{d^{2} \mathbf{r}}{8 \pi} \operatorname{Tr}\left[\nabla \hat{Q}^{\dagger} \cdot \nabla \hat{Q}\right]+k S_{\mathrm{WZNW}},
\end{aligned}
$$

where $\hat{Q}$ is an $\mathrm{SU}(n)$ group element acting on the fundamental representation of the replica space, $S_{\mathrm{WZNW}}$ is the WessZumino-Novikov-Witten term [Eq. (2.39)], and $\phi$ denotes the free boson field [Abelian bosonization of the spin U(1) sector].

The appearance of the Abelian disorder parameter $\lambda_{A}$ in Eq. (3.1) can be understood as follows. Even for $\lambda_{A}=0$, the free boson $\phi$ still plays an important role, in that holomorphic primary fields of the parent $\mathrm{U}(n k)_{1}$ theory are products of vertex operators : $\exp ( \pm i \beta \varphi)$ : and primary fields from the $\mathrm{SU}(n)_{k}$ and $\mathrm{SU}(k)_{n}$ replica and valley constituents [20]. Here, $\varphi$ denotes the holomorphic component of $\phi$,

$$
\phi(\mathbf{r})=\varphi(z)+\bar{\varphi}(\bar{z}) .
$$

For example, the holomorphic free fermion field $L$ in Eq. (2.15b) can be understood (roughly) [57] as the product

$$
L_{v, a}(z)=: \exp [i \sqrt{4 \pi} \varphi(z)]: \Omega_{1, a}^{(\mathrm{R})}(z) \Omega_{1, v}^{(\mathrm{V})}(z),
$$

where $\Omega_{1}^{(\mathrm{R})}(z)$ and $\Omega_{1}^{(\mathrm{V})}(z)$ denote the primary fields in the fundamental representation of the replica $\operatorname{SU}(n)_{k}$ and valley $\mathrm{SU}(k)_{n}$ sectors, respectively. In the clean theory [Eq. (2.13) with $\mathbf{A}_{i}=\mathbf{A}=0$ ], $L(z)$ has the holomorphic dimension [45]

$$
\begin{aligned}
h_{1} & \equiv h_{1}^{[\mathrm{U}(1)]}+h_{1}^{\left[\mathrm{SU}(n)_{k}\right]}+h_{1}^{\left[\mathrm{SU}(k)_{n}\right]} \\
& =\frac{1}{2 n k}+\frac{n^{2}-1}{2 n(n+k)}+\frac{k^{2}-1}{2 k(n+k)}=\frac{1}{2} .
\end{aligned}
$$

Here, we have used the normalization of the $\phi$ action in Eq. (3.1) with $\lambda_{A}=0$.

The Abelian vector potential A in Eq. (2.13) couples to the spin $\mathrm{U}(1)$ current $\mathbf{J}$. With our normalization, this bosonizes to $(n k / \sqrt{4 \pi}) i \nabla \phi$. Averaging over the disorder gives the $\lambda_{A}$ term in Eq. (3.1).

In the presence of disorder, the $\mathrm{SU}(k)_{n}$ sector "localizes." As a result, the dimension of $L(z)$ in the low-energy $\mathrm{U}(n)_{k}$ CFT is reduced to

$$
h_{1}=\frac{1}{2 n k\left(1+\lambda_{A} n k\right)}+\frac{n^{2}-1}{2 n(n+k)} .
$$

The LDoS operator is $R_{a}^{\dagger} L_{a}+L_{a}^{\dagger} R_{a}$, which has dimension

$$
x_{1}=2 h_{1}=\frac{1}{k^{2}}-\lambda_{A},
$$

where we have taken the replica $n \rightarrow 0$ limit. This result was originally obtained in Ref. [20].

Higher LDoS moments are not conformal group eigenoperators. However, the $q$ th $\operatorname{LDoS}$ moment has a most relevant component that corresponds to the primary field with weight $\omega_{q}$, which is the completely antisymmetric tensor representation with $q$ indices. Here, the symbol $\omega_{p}, p \in$ $\{1,2, \ldots, r\}$ denotes the $p$ th fundamental weight for a rank $r$ Lie algebra [45]. As a result, the $q$ th moment has the scaling dimension

$$
\begin{aligned}
x_{q} & =2\left(h_{q}^{[\mathrm{U}(1)]}+h_{q}^{\left[\mathrm{SU}(n)_{k}\right]}\right) \\
& =q-\left(\frac{k-1}{k^{2}}\right)\left(k q+q^{2}\right)-\lambda_{A} q^{2},
\end{aligned}
$$

where we have taken the replica limit. In Eq. (3.6), for nonzero $n$ one has

$$
h_{q}^{[\mathrm{U}(1)]}=\frac{q^{2}}{2 n k\left(1+\lambda_{A} n k\right)}, \quad h_{q}^{\left[\mathrm{SU}(n)_{k}\right]}=\frac{q(n-q)(n+1)}{2 n(n+k)} .
$$

Equation (3.6) was originally obtained in Refs. [32,33]. The class AIII multifractal spectrum in Eqs. (2.35) and (2.36) obtains from combining Eqs. (2.34) and (3.6).

\section{Interaction operators}

We work in the language of the holomorphic decomposition in Eq. (2.15). A generic rotationally invariant, spin U(1) invariant interaction can be expressed as a linear combination of

$$
\begin{aligned}
& \mathcal{O}_{a, v_{1} v_{3}}^{(\mathrm{A}) v_{2} v_{4}} \equiv R_{v_{1}, a}(\bar{z}) R^{\dagger v_{2}, a}(\bar{z}) L_{v_{3}, a}(z) L^{\dagger v_{4}, a}(z), \\
& \mathcal{O}_{a, v_{3} v_{4}}^{\text {(B) } v_{1} v_{2}} \equiv R^{\dagger v_{1}, a}(\bar{z}) R^{\dagger v_{2}, a}(\bar{z}) L_{v_{3}, a}(z) L_{v_{4}, a}(z), \\
& \mathcal{O}_{a, v_{1} v_{2}}^{(\mathrm{C}) v_{3} v_{4}} \equiv R_{v_{1}, a}(\bar{z}) R_{v_{2}, a}(\bar{z}) L^{\dagger v_{3}, a}(z) L^{\dagger v_{4}, a}(z) .
\end{aligned}
$$

Here and in what follows, no sum on the replica index $a$ is implied.

a. Interaction type A. We consider first $\mathcal{O}_{a}^{(\mathrm{A})}$, with holomorphic component $L_{v, a}(z) L^{\dagger v^{\prime}, a}(z)$. The traceless, replicaresolved tensor

$$
L_{v, a} L^{\dagger v^{\prime}, a}-\frac{1}{k} \delta_{v}^{v^{\prime}} \sum_{v^{\prime \prime}} L_{v^{\prime \prime}, a} L^{\dagger v^{\prime \prime}, a}
$$

transforms in the adjoint representation of the valley $\mathrm{SU}(k)$; because it is replica-resolved, this bilinear cannot be a valley Kac-Moody current. For $\mathrm{SU}(N)_{q}$ with $q \geqslant 2$, the adjoint representation can be associated to a primary field with the affine weight $(q-2) \hat{\omega}_{0}+\hat{\omega}_{1}+\hat{\omega}_{N-1}$, with holomorphic dimension

$$
h_{(10 \cdots 01)}=\frac{N}{N+q} .
$$

[The indices in the $(\cdots)$ subscript denote (nonaffine) Dynkin coefficients.] The product of $\mathrm{SU}(n)_{k}$ and $\mathrm{SU}(k)_{n}$ adjoint representation primary fields has the holomorphic dimension

$$
\frac{n}{n+k}+\frac{k}{n+k}=1,
$$

as expected. In the conformal remnant theory $\mathrm{U}(1) \oplus \mathrm{SU}(n)_{k}$ [Eq. (1.1)], the corresponding interaction operator (diagonal primary field $\equiv \mathcal{O}_{a}^{(\mathrm{A}, 1)}$ ) carries scaling dimension $x_{2}^{(\mathrm{A}, 1)}=0$ in 
the replica $n \rightarrow 0$ limit. We can write this interaction operator as

$$
\mathcal{O}_{a}^{(\mathrm{A}, 1)} \equiv 2 J_{\kappa a}^{i} \bar{J}_{\kappa a}^{i}, \quad x_{2}^{(\mathrm{A}, 1)}=0
$$

where

$$
J_{\kappa a}^{i} \equiv L^{\dagger a} \hat{t}_{\kappa}^{i} L_{a}, \quad \bar{J}_{\kappa a}^{i} \equiv R^{\dagger a} \hat{t}_{\kappa}^{i} R_{a}
$$

denote replica-resolved valley $\mathrm{SU}(k)$ currents.

We also define the valley singlet operators

$$
J_{a} \equiv \sum_{v} L^{\dagger v, a} L_{v, a}, \quad \bar{J}_{a} \equiv \sum_{v} R^{\dagger v, a} R_{v, a} .
$$

These are replica Kac-Moody currents. The corresponding interaction operator has dimension 2 . We denote this as

$$
\mathcal{O}_{a}^{(\mathrm{A}, 2)} \equiv J_{a} \bar{J}_{a}, \quad x_{2}^{(\mathrm{A}, 2)}=2 .
$$

b. Interaction types $\mathrm{B}$ and $\mathrm{C}$. We consider next $\mathcal{O}_{a}^{(\mathrm{B})}$. The relevant holomorphic bilinear is $L_{v_{3}, a}(z) L_{v_{4}, a}(z)$. This must be antisymmetrized in valley indices (due to Fermi statistics), while the replica indices belong to the symmetric representation. The relevant affine weight for the antisymmetric (symmetric) representation is $(q-1) \hat{\omega}_{0}+\hat{\omega}_{2}\left[(q-2) \hat{\omega}_{0}+\right.$ $\left.2 \hat{\omega}_{1}\right]$. In $\operatorname{SU}(N)_{q}$,

$$
\begin{aligned}
& h_{(010 \cdots 0)}=\frac{(N-2)(N+1)}{N(N+q)}, \\
& h_{(200 \cdots 0)}=\frac{(N+2)(N-1)}{N(N+q)} .
\end{aligned}
$$

For $\lambda_{A}=0$, the product of $\mathrm{U}(1),(200 \cdots 0) \mathrm{SU}(n)_{k}$, and $(010 \cdots 0) \mathrm{SU}(k)_{n}$ primary fields must carry conformal dimension $h=1$. This determines the $U(1)$ dimension $h^{[\mathrm{U}(1)]}$ via

$$
\left.h^{[\mathrm{U}(1)]}\right|_{\lambda_{A}=0}=1-h_{(200 \cdots 0)}^{\left[\mathrm{SU}(n)_{k}\right]}-h_{(010 \cdots 0)}^{\left[\mathrm{SU}(k)_{n}\right]}=\frac{2}{n k} .
$$

Restoring $\lambda_{A}>0$, the conformal dimension of the interaction in the $\mathrm{U}(1) \oplus \mathrm{SU}(n)_{k}$ remnant theory is

$$
h^{(\mathrm{B})}=\frac{2}{n k\left(1+\lambda_{A} n k\right)}+\frac{(n+2)(n-1)}{n(n+k)} .
$$

We can write the corresponding diagonal interaction operator as

$$
\mathcal{O}_{a}^{(\mathrm{B})} \equiv I_{\left[v_{1} v_{2}\right] a} \bar{I}^{\dagger\left[v_{2} v_{1}\right] a}, \quad x_{2}^{(\mathrm{B})}=\frac{2(k+2)}{k^{2}}-4 \lambda_{A},
$$

where we have taken the replica limit of twice Eq. (3.15), and where we have defined

$$
I_{\left[v_{1} v_{2}\right] a} \equiv L_{v_{1}, a} L_{v_{2}, a}, \quad \bar{I}^{\dagger\left[v_{1} v_{2}\right] a} \equiv R^{\dagger v_{1}, a} R^{\dagger v_{2}, a} .
$$

Interaction $\mathcal{O}_{a}^{(\mathrm{C})}$ carries the same scaling dimension, and is defined via

$$
\mathcal{O}_{a}^{(\mathrm{C})} \equiv I^{\dagger\left[v_{1} v_{2}\right] a} \bar{I}_{\left[v_{2} v_{1}\right] a}, \quad x_{2}^{(\mathrm{C})}=\frac{2(k+2)}{k^{2}}-4 \lambda_{A},
$$

where

$$
I^{\dagger\left[v_{1} v_{2}\right] a} \equiv L^{\dagger v_{1}, a} L^{\dagger v_{2}, a}, \quad \bar{I}_{\left[v_{1} v_{2}\right] a} \equiv R_{v_{1}, a} R_{v_{2}, a} .
$$

c. AIII interactions and Fierz identities. We write the interaction Hamiltonian as a generic Hermitian linear combination of the operators in Eqs. (3.9), (3.12), (3.16), and (3.18):

$$
H_{\mathrm{AIII}}^{(I)}=\sum_{a} \int d^{2} \mathbf{r}\left[U \mathcal{O}_{a}^{(\mathrm{A}, 1)}+V \mathcal{O}_{a}^{(\mathrm{A}, 2)}+W\left(\mathcal{O}_{a}^{(\mathrm{B})}+\mathcal{O}_{a}^{(\mathrm{C})}\right)\right] .
$$

We exploit the $\mathrm{SU}(k)$ Fierz identity in Table I to rewrite

$$
\mathcal{O}_{a}^{(\mathrm{A}, 1)}=-2\left(L_{a}^{\dagger} R_{a}\right)\left(R_{a}^{\dagger} L_{a}\right)-\frac{2}{k} J_{a} \bar{J}_{a} .
$$

We also have

$$
\mathcal{O}_{a}^{(\mathrm{B})}=\left(R_{a}^{\dagger} L_{a}\right)^{2}, \quad \mathcal{O}_{a}^{(\mathrm{C})}=\left(L_{a}^{\dagger} R_{a}\right)^{2} .
$$

Clearly, for the case of a single species ("valley") $k=1$, $\mathcal{O}_{a}^{(\mathrm{A}, 1)}=\mathcal{O}_{a}^{(\mathrm{B})}=\mathcal{O}_{a}^{(\mathrm{C})}=0$.

The spin density $S^{z}$ and the spin singlet, valley singlet mass operator $m$ were defined in Eqs. (2.5) and (2.22). Expressing these via the holomorphic decomposition in Eq. (2.14) gives

$$
\begin{aligned}
S_{a}^{z} & =\frac{1}{2}\left(R_{a}^{\dagger} L_{a}+L_{a}^{\dagger} R_{a}\right), \\
m_{a} & =R_{a}^{\dagger} L_{a}-L_{a}^{\dagger} R_{a}, \\
\Rightarrow R_{a}^{\dagger} L_{a} & =S_{a}^{z}+\frac{1}{2} m_{a}, \quad L_{a}^{\dagger} R_{a}=S_{a}^{z}-\frac{1}{2} m_{a} .
\end{aligned}
$$

We then arrive at

$$
\begin{aligned}
\mathcal{O}_{a}^{(\mathrm{A}, 1)} & =\frac{1}{2}\left(m_{a} m_{a}-4 S_{a}^{z} S_{a}^{z}\right)-\frac{2}{k} J_{a} \bar{J}_{a}, \\
\mathcal{O}_{a}^{(\mathrm{B})}+\mathcal{O}_{a}^{(\mathrm{C})} & =\frac{1}{2}\left(m_{a} m_{a}+4 S_{a}^{z} S_{a}^{z}\right) .
\end{aligned}
$$

Using Eqs. (3.12) and (3.24) in Eq. (3.20), we recover the form of the class AIII interaction Hamiltonian in Eq. (2.59).

The "tree level" class AIII RG flows in Eq. (2.61) obtain from inserting $x_{1}$ from Eq. (3.5) and the interaction dimensions from Eqs. (3.9), (3.12), (3.16), and (3.18) into Eq. (2.47).

\section{B. Class DIII}

\section{Density of states and multifractal spectrum}

In class DIII, the effective CFT is $\mathrm{SO}(n)_{k}$ [Eq. (1.1)]. In fact, it is convenient to consider $2 n$ replicas, so that we work with the affine version of the $D_{n}$ Lie algebra. As the results for LDoS scaling dimensions are new to our knowledge, we provide more details than we did in Sec. III A 1, above.

We will denote the (regular, nonaffine) fundamental weights of the algebra as $\omega_{p}, p \in\{1,2, \ldots, n\}$. Affine weights will be denoted with a "hat," $\hat{\omega}_{m}, m \in\{0,1,2, \ldots, n\}$. The following facts about the quadratic form matrix [45] $F_{i, j} \equiv\left\langle\omega_{i}, \omega_{j}\right\rangle$ are useful:

$$
\begin{aligned}
F_{i, j} & =\min (i, j), \quad 1 \leqslant\{i, j\} \leqslant n-2, \\
F_{i, n-1} & =F_{i, n}=\frac{i}{2}, \quad 1 \leqslant i \leqslant n-2, \\
F_{n-1, n} & =\frac{n-2}{4}, \quad F_{n-1, n-1}=F_{n, n}=\frac{n}{4} .
\end{aligned}
$$

The "outer" $\left[(n-1)^{\text {th }}\right.$ and $\left.n^{\text {th }}\right]$ rows and columns reflect the two spinor representations. These results are most easily obtained using the expansion of the fundamental weights 
in terms of the orthonormal weights of the fundamental representation.

We consider first the minimal non-Abelian case with $k=3$ valleys. At level one (free Majorana fermions), only the $\omega_{1}$, $\omega_{n-1}$ and $\omega_{n}$ representations correspond to primary fields. At level $k=2$, the full set consists of the affine weights

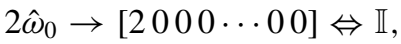

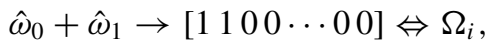

$$
\begin{aligned}
& \hat{\omega}_{0}+\hat{\omega}_{n-1} \rightarrow\left[\begin{array}{llllll}
1 & 0 & 0 & 0 & \cdots & \cdots
\end{array}\right] \Leftrightarrow \Omega_{\sigma}, \\
& \hat{\omega}_{0}+\hat{\omega}_{n} \rightarrow\left[\begin{array}{lllllll}
1 & 0 & 0 & 0 & \cdots & 0 & 1
\end{array}\right] \Leftrightarrow \Omega_{\bar{\sigma}}, \\
& 2 \hat{\omega}_{1} \rightarrow\left[\begin{array}{llllll}
0 & 2 & 0 & 0 & \cdots & 0
\end{array}\right] \Leftrightarrow \tilde{\Omega}_{(i j)},
\end{aligned}
$$

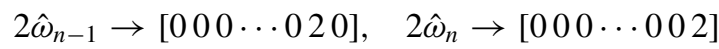

$$
\begin{aligned}
& \Leftrightarrow \Omega_{\left[i_{1} i_{2} \cdots i_{n}\right]}, \\
& \hat{\omega}_{1}+\hat{\omega}_{n-1} \rightarrow\left[\begin{array}{llllll}
0 & 1 & 0 & 0 & \cdots 1 & 0
\end{array}\right] \Leftrightarrow \Omega_{i \sigma}
\end{aligned}
$$

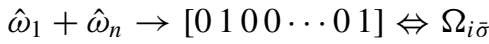

$$
\begin{aligned}
& \hat{\omega}_{n-1}+\hat{\omega}_{n} \rightarrow[00000 \cdots 11] \Leftrightarrow \Omega_{\left[i_{1} i_{2} \cdots i_{n-1}\right]},
\end{aligned}
$$



$$
\begin{aligned}
& \hat{\omega}_{3} \rightarrow\left[\begin{array}{lllllll}
0 & 0 & 0 & 1 & \cdots & 0 & 0
\end{array}\right] \Leftrightarrow \Omega_{[i j k]}, \\
& \hat{\omega}_{n-2} \rightarrow\left[\begin{array}{llllll}
0 & 0 & 0 & \cdots & 0 & 0
\end{array}\right] \Leftrightarrow \Omega_{\left[i_{1} i_{2} \cdots i_{n-2}\right]} .
\end{aligned}
$$

Here, the numbers in square brackets are affine Dynkin coefficients. The affine weight $\hat{\omega}_{0}$ is the basic fundamental weight (i.e., the vacuum for $k=1$ ). The $\Omega$ 's denote the associated holomorphic primary fields, irreducible tensors with indices that transform in some combination of fundamental replica $(i, j \in\{1,2, \ldots, 2 n\})$ and spinor $\left(\sigma, \bar{\sigma} \in\left\{1,2, \ldots, 2^{n-1}\right\}\right)$ representations. In particular, $\Omega_{\left[i_{1} i_{2} \cdots i_{p}\right]}$ is a fully antisymmetric rank- $p$ tensor, while $\tilde{\Omega}_{(i j)}$ is the traceless symmetric 2 nd rank tensor.

All of the fields in Eq. (3.26) appear as primaries at level $k=3$, since we can add $\hat{\omega}_{0}$ to each. Additional dominant weights at level 3 are the unique triple sums of $\left\{\hat{\omega}_{1}, \hat{\omega}_{n-1}, \hat{\omega}_{n}\right\}$, and the sum of one of these with one of the level 2 weights $\left\{\hat{\omega}_{2}, \hat{\omega}_{3}, \ldots, \hat{\omega}_{n-2}\right\}$. In particular, we get mixed tensors such as

$$
\begin{aligned}
\hat{\omega}_{1}+\hat{\omega}_{i} & \rightarrow\left[\begin{array}{llllll}
0 & 1 & 0 & 0 & \cdots 0 & i^{\text {th }} \text { place } \\
1 & 0 \cdots 0
\end{array}\right] \\
& \Rightarrow \Omega_{j^{\prime}\left[j_{1} j_{2} \cdots j_{i}\right]}, \quad i \in\{2,3, \ldots, n-2\} .
\end{aligned}
$$

The weights $\hat{\omega}_{n-1}+\hat{\omega}_{i}$ and $\hat{\omega}_{n}+\hat{\omega}_{i}$ belong to the conjugacy classes of the spinor representations.

The $q$ th LDoS moment involves exactly $q$ distinct replica labels (no more, no less). Therefore, this should be associated to the most relevant component of the decomposition of a generic tensor $T_{i_{1} i_{2} \cdots i_{q}}(q \ll 2 n)$. This rules out the basic spinor (twist field) representations $\hat{\omega}_{n-1}$ and $\hat{\omega}_{n}$, and all fields in their conjugacy classes. At level $k=3$, for the $q$ th moment there are only two possibilities:

$$
\hat{\omega}_{0}+\hat{\omega}_{q}: \quad h_{\omega_{q}}=\frac{\left\langle\omega_{q}, \omega_{q}+2 \rho\right\rangle}{2(k+g)}=\frac{F_{q, q}+2 \sum_{i=1}^{n} F_{q, i}}{2(k+2 n-2)}=\frac{(2 n-q) q}{2(k+2 n-2)} .
$$

Note that we recover the free fermion dimension $h=1 / 2$ for $k=q=1$ (and any $n$ ),

$$
\hat{\omega}_{1}+\hat{\omega}_{q-1}: \quad h_{\omega_{1}+\omega_{q-1}}=\frac{\left[F_{q-1, q-1}+2 F_{q-1,1}+F_{1,1}+2 \sum_{i=1}^{n}\left(F_{1, i}+F_{q-1, i}\right)\right]}{2(k+2 n-2)}=\frac{(2+2 n-q) q}{2(k+2 n-2)} .
$$

We see that the antisymmetric tensor $\Omega_{\left[i_{1} \cdots i_{q}\right]} \Leftrightarrow \omega_{q}$ corresponds to the most negative scaling dimension in the replica $n \rightarrow 0$ limit. The same applies in classes AIII and CI, and holds for larger $k$ as well.

Thus the scaling dimension of the $q$ th LDoS moment is given by

$$
x_{q}=2 h_{\omega_{q}}=-\frac{q^{2}}{(k-2)},
$$

where we have taken the replica limit. The class DIII multifractal spectrum in Eq. (2.35) and (2.36) obtains from combining Eqs. (2.34) and (3.30).

We note that the $k=3$ case corresponds to quite strong multifractality: the critical $q$ for multifractal spectral termination [24-26,81] is given by

$$
q_{c}=\sqrt{2(k-2)}=\sqrt{2} .
$$

Thus, unlike the minimal non-Abelian realizations of classes CI and AIII, the second moment is already beyond termination. For $k=3$, this potentially complicates the treatment of interactions (four-fermion operators), because annealed (disorder) and quenched (spatial) averages are no longer the same [24,81]. However, it turns out that interaction operator scaling dimension $x_{2}^{(U)}=0>x_{2}$ (Secs. II D 3 and III B 2), so that this issue does not arise.

\section{Interaction operator}

The mass-squared (BCS pairing) interaction operator in Eq. (2.71) can be expressed through the decomposition in Eq. (2.20) as

$$
m_{a} m_{a}=-4 L_{v_{1}, a} R_{v_{1}, a} L_{v_{2}, a} R_{v_{2}, a}=-2 J_{\kappa a}^{i} \bar{J}_{\kappa a}^{i},
$$

where the replica-resolved valley $\mathrm{SO}(k)$ currents are defined via

$$
J_{\kappa a}^{i} \equiv L_{a} \hat{\mathfrak{t}}_{\kappa}^{i} L_{a}, \quad \bar{J}_{\kappa a}^{i} \equiv R_{a} \hat{\mathfrak{t}}_{\kappa}^{i} R_{a},
$$

and we have used the Fierz identity in Table I. The holomorphic field $J_{\kappa a}^{i}$ transforms in the antisymmetric adjoint representation $\left(\omega_{2}\right)$ in valley space, and the traceless symmetric representation $\left(2 \omega_{1}\right)$ in replica space. Here, $\omega_{p}$ denotes the $p$ th (nonaffine) fundamental weight. In the remnant $\mathrm{SO}(2 n)_{k}$ 
theory, this has the holomorphic dimension

$$
h_{(200 \cdots 0)}=\frac{4 F_{1,1}+4 \sum_{i=1}^{n} F_{1, i}}{2(k+2 n-2)}=\frac{2 n}{(k+2 n-2)} .
$$

As a check, for $k$ even, the dimension of the antisymmetric representation in the $\mathrm{SO}(k)_{2 n}$ theory is [Eq. (3.28)]

$$
h_{(010 \cdots 0)}=\frac{2(k-2)}{2(k+2 n-2)},
$$

so that $h_{(200 \cdots 0)}+h_{(010 \cdots 0)}=1$. Taking the replica limit of Eq. (3.34) gives $x_{2}^{(U)}=0$ for the interaction in Eq. (2.71). The flow equation (2.72) then obtains from Eq. (2.47) and (3.30) with $q=1$.

\section{CONCLUSIONS AND OPEN QUESTIONS}

In summary, surface states of topological superconductors (TSCs) are critically delocalized in the absence of interactions, with wave function statistics determined exactly by conformal field theory (CFT) $[15,19,20,24,32-36]$. Clean surface states are robust against sufficiently weak interactions, owing to the vanishing density of states at the Majorana surface band Dirac point.

The main result of this paper is that the combination of both weak disorder and weak interactions is more subtle than either in isolation. Wave-function multifractality [22,23] (spatial inhomogeneity) and Chalker scaling [37-39] (correlations between the spatial structures of different wave functions) can amplify interparticle interactions [15,40,41]. For class CI, the effect is so strong that we predict the absence of gapless surface states, as the multifractal enhancement of interaction matrix elements destabilizes the noninteracting fixed point [15]. The Wess-Zumino-Novikov-Witten [91] Finkel'stein nonlinear sigma model [46,47] (WZNW-FNLsM) does not reveal the existence of a perturbatively accessible critical fixed point, and the most likely scenario is that time-reversal symmetry always breaks spontaneously. The dominant instability realizes the (class $\mathrm{C}$ ) spin quantum Hall effect at the surface $[4,15,74]$.

By contrast, stable surface states in the presence of both disorder and interactions are possible in classes AIII and DIII. For a class AIII, topological superconductor with a winding number modulus $|\nu| \geqslant 2$, CFT predicts a window of disorder strengths in which interactions are irrelevant. By combining the CFT scaling dimensions with a phenomenological BCS term that favors pairing of the surface quasiparticles, we predict interaction-stabilized critical fixed points outside of this range. For $|\nu| \gg 1$, this agrees with the one-loop result of the WZNW-FNLsM, which is well-controlled in this limit. The stability of these nontrivial fixed points to higher order loop corrections is an important topic for future work, as is the characterization of observables.

In class DIII, CFT predicts that interactions are always irrelevant. This result obtains despite the fact that the disorderaveraged density of states diverges near zero energy in class DIII, for $|v| \geqslant 3$. The key is that there is no multifractal enhancement of the interaction matrix elements in this case, due to the absence of a continuous symmetry in the presence of disorder. The surface states of both classes AIII and DIII can be gapped by sufficiently strong interactions, leading to spontaneous time-reversal symmetry breaking. The instabilities are expected to produce surface spin or thermal quantum Hall states [72,73].

In the absence of interactions, the dc zero temperature spin conductance is universal at the surface of topological superconductors in classes CI and AIII [19,30,64]. We argued that the surface thermal conductance in class DIII is also universal. We discussed (but did not derive) the result that the lowest-order Altshuler-Aronov interaction conductance corrections vanish in the conformal limit. We derive and examine this point in detail elsewhere [51]. The full one-loop WZNW-FNLsM RG equations are also derived in that work.

In Ref. [61], we verified CFT predictions for noninteracting surface states with two valleys in classes CI and AIII using numerical methods. In that work, we considered the average density of states and the multifractal spectrum of local density of states fluctuations. To our knowledge, transport has not been simulated in these models. Class DIII for $|\nu| \geqslant 3$ remains open for numerical investigation. In particular, we argued here that the thermal conductance is universal, and that this is reflected by the topological protection of conformal invariance. CFT gives universal predictions for multifractal spectra and the dynamic critical exponent. On the other hand, it is known that one can realize a diffusive "thermal metal" phase in class DIII [80]. While our arguments suggest that this does not occur at the surface of a topological superconductor, it would be interesting to verify this result with numerics. Another nontrivial check would be to study surface states in disordered 3D lattice models of bulk TSCs.

\section{ACKNOWLEDGMENTS}

We thank V. Kravtsov, M. Mueller, A. Scardicchio, I. Gornyi, I. Gruzberg, and A. Mirlin for helpful discussions. This work was supported by the Welch Foundation under Grant No. C-1809.

\section{APPENDIX A: NONVALLEY INVARIANT MASS OPERATORS: CLASS CI}

In addition to the unique valley- and spin-symmetric mass operator defined in Eq. (2.22), there are other Dirac mass operators that do not preserve valley and/or spin symmetry. These also break time-reversal invariance. The noninvariant mass operators can be organized into different irreducible representations of the valley and spin symmetry groups. In class CI, for example, there are two such classes of operators:

$$
\begin{aligned}
M^{a}(\mathbf{r}) & \equiv \psi^{\dagger} \hat{\sigma}^{3} \hat{\mathfrak{a}}_{\mu}^{a} \hat{\kappa}^{\mu} \psi, \\
\mathscr{M}^{n, i}(\mathbf{r}) & \equiv \mathrm{L} \hat{s}^{n} \hat{\mathfrak{t}}_{\kappa}^{i} \mathcal{R} .
\end{aligned}
$$

In Eq. (A1a), $\mu \in\{0,1,2,3\}$ and $\hat{\kappa}^{\mu}=\left\{\mathbb{I}, \hat{\kappa}^{1,2,3}\right\}$ are the $2 k \times$ $2 k$ block identity and Pauli matrices in valley space. The latter commute with the $k \times k$ matrices $\hat{\mathfrak{a}}_{\mu}^{a}$, which satisfy

$$
\operatorname{Tr}\left[\hat{\mathfrak{a}}_{0}^{a}\right]=0, \quad\left(\hat{\mathfrak{a}}_{0}^{a}\right)^{\top}=\hat{\mathfrak{a}}_{0}^{a}, \quad\left(\hat{\mathfrak{a}}_{1,2,3}^{a}\right)^{\top}=-\hat{\mathfrak{a}}_{1,2,3}^{a} .
$$

We have expressed $\mathscr{M}^{n, i}$ in terms of the decomposition in Eq. (2.6); $\hat{s}^{n}$ denotes a spin space Pauli matrix.

The mass operator $M^{a}$ is spin $\mathrm{SU}(2)$ invariant, and transforms under the $\omega_{2}$ (antisymmetric second rank tensor) 
representation of the valley $\mathrm{Sp}(2 k)$ symmetry group. (Here, $\omega_{2}$ is the second fundamental weight [45].) A nonzero $\left\langle M^{a}\right\rangle$ allows the realization of spin Hall plateaux with $k$ replaced by any $p \in\{-k,-k+2, \ldots, k-2, k\}$ in Eq. (2.23).

The mass operator $\mathscr{M}^{n, i}$ transforms in the adjoint representations of the spin $\mathrm{SU}(2)$ and valley $\mathrm{Sp}(2 k)$ symmetries. A nonzero average of this operator would imply broken spin symmetry, and the resulting surface quantum Hall state will reside in either class $A$ [residual spin $U(1)$ invariance] or class $\mathrm{D}$ (no spin symmetry).

\section{APPENDIX B: FLOW EQUATION FOR $\lambda_{A}$ IN CLASS AIII AWAY FROM THE CONFORMAL FIXED POINT}

In the absence of interparticle interactions, the action for the $\mathrm{U}(1) \oplus \mathrm{SU}(n)_{k}$ class AIII low-energy effective field theory is transcribed in Eq. (3.1), above. For a nontopological (Gade) class AIII disordered quantum system in $2 \mathrm{D}$, the replica theory for quantum diffusion (principal chiral model) is

$$
\begin{aligned}
S_{\mathrm{AIII}}^{(\mathrm{PCM})}= & \frac{1}{\lambda} \int \frac{d^{2} \mathbf{r}}{8 \pi} \operatorname{Tr}\left[\nabla \hat{U}^{\dagger} \cdot \nabla \hat{U}\right] \\
& -\frac{\lambda_{A}}{\lambda^{2}} \int \frac{d^{2} \mathbf{r}}{8 \pi}\left[\operatorname{Tr}\left(\hat{U}^{\dagger} \nabla \hat{U}\right)\right]^{2},
\end{aligned}
$$

where $\hat{U}$ is a $\mathrm{U}(n)$-valued matrix field. We write

$$
\hat{U} \equiv \exp (i \phi) \hat{Q},
$$

and thereby obtain

$$
\begin{aligned}
S_{\mathrm{AIII}}^{(\mathrm{PCM})}= & \frac{n}{\lambda}\left(1+\frac{n \lambda_{A}}{\lambda}\right) \int \frac{d^{2} \mathbf{r}}{8 \pi} \nabla \phi \cdot \nabla \phi \\
& +\frac{1}{\lambda} \int \frac{d^{2} \mathbf{r}}{8 \pi} \operatorname{Tr}\left[\nabla \hat{Q}^{\dagger} \cdot \nabla \hat{Q}\right] .
\end{aligned}
$$

The coupling strength of the U(1) term (free boson) cannot be renormalized, so that

$$
\frac{d \lambda_{A}}{d l}=\left(\frac{1}{n}+2 \frac{\lambda_{A}}{\lambda}\right) \frac{d \lambda}{d l} .
$$

Equation (B1) is a consistent deformation of the AIII model away from $\lambda=1 / k$ [Eq. (2.38)], except that it is missing the Wess-Zumino-Novikov-Witten term. Incorporating the latter, the one-loop beta equation for $\lambda$ is given by

$$
\frac{d \lambda}{d l}=n \lambda^{2}\left[1-(k \lambda)^{2}\right]+\cdots,
$$

where we have not yet taken the replica $n \rightarrow 0$ limit. Combining Eqs. (B3) and (B4) leads to

$$
\frac{d \lambda_{A}}{d l}=\lambda^{2}\left[1-(k \lambda)^{2}\right] .
$$

Equation (B5) was first obtained in Ref. [92], wherein the authors claimed that this is the exact beta function for the perturbation $\lambda \neq 1 / k$ of the class AIII CFT, in the absence of interactions. It implies that (1) $\lambda_{A}$ is always generated under the RG except at the WZNW point $\lambda=1 / k$, and (2) $\lambda_{A}$ is driven to arbitrarily positive (negative) values for $\lambda<1 / k$ $(\lambda>1 / k)$.

\section{APPENDIX C: TREE LEVEL RG FOR INTERACTIONS AT A CRITICALLY DELOCALIZED FIXED POINT}

In this appendix, we derive Eq. (2.47). Consider the class CI theory in Eq. (2.1). In a fixed realization of the disorder, we can write a $(2+1)$-D zero-temperature imaginary time path integral with the action

$$
\begin{aligned}
S= & \int d \tau d^{2} \mathbf{r} \bar{\psi}\left\{\eta \partial_{\tau}+\frac{1}{2 \pi}\left[\hat{\boldsymbol{\sigma}} \cdot\left(i \nabla-\mathbf{A}_{i} \hat{\mathfrak{t}}_{\kappa}^{i}\right)\right]\right\} \psi \\
& +U \sum_{a} T_{\left\{v_{i}\right\}}^{\left\{\sigma_{i}\right\}} \int d \tau d^{2} \mathbf{r} \bar{\psi}_{\sigma_{1}, v_{1}, a} \bar{\psi}_{\sigma_{2}, v_{2}, a} \psi_{\sigma_{3}, v_{3}, a} \psi_{\sigma_{4}, v_{4}, a},
\end{aligned}
$$

where we have included a generic four-fermion interaction with coupling strength $U$. Here, $a$ denotes the replica index, while the pseudospin-valley tensor $T_{\left\{v_{i}\right\}}^{\left\{\sigma_{i}\right\}}$ is chosen such that the interaction respects the defining class CI symmetries [time reversal and spin SU(2) invariances]. The parameter $\eta$ multiplying the imaginary time derivative is necessary to keep track of the dynamic critical exponent, i.e., the fact that time and space need not scale in the same way in the disorder-averaged theory. The bare value of $\eta=1$, but under renormalization this parameter will flow. We use a renormalization group (RG) scheme that fixes the coefficient of the kinetic term $\bar{\psi} i \hat{\boldsymbol{\sigma}} \cdot \nabla \psi$. An alternative would be to fix the $\bar{\psi} \partial_{\tau} \psi$ term and renormalize the Fermi velocity [99]. This is, however, undesirable here, as it will lead to a renormalization of the spatial components of the stress tensor. We require a fixed normalization of the latter to determine scaling dimensions using $(2+0)$-D conformal field theory [45].

We assume that the imaginary time variable $\tau$ carries units of $L^{z}$, where $L$ denotes the system size and $z$ is a (possibly scale-dependent) "dynamic critical exponent." In inverse length units, one then has the dimensions

$$
[\psi(\tau, \mathbf{r})]=\frac{1+z}{2}, \quad[\eta]=1-z, \quad[U]=-z .
$$

The tree level RG equation for $U$ is then given by

$$
\frac{d \ln U}{d l}=-z+\left(\frac{d \ln U}{d l}\right)_{a}
$$

where the second term is the "anomalous" dimension due to renormalization by the disorder.

Averaging over disorder as in Eq. (2.24) induces an effective four-fermion disorder vertex. The renormalization of $U$ due to the disorder involves an infinite sum over diagrams with one interaction vertex and any number of disorder vertices. Because the disorder is static, these diagrams do not involve integrals over Matsubara frequencies; the ultraviolet divergences obtain exclusively from momentum integrations. Given the strong correlations in spatial structure implied by Chalker scaling [37-39] for the low-energy wave functions, it is sufficient to determine the renormalization of the interaction matrix elements in an effective $(2+0)$-D theory. This can be understood as the projection of Eq. (C1) to zero Matsubara frequency for all fermion fields. In two spatial dimensions, a four-fermion term has engineering dimension equal to two; 
therefore

$$
\left(\frac{d \ln U}{d l}\right)_{a}=2-x_{2}^{(U)},
$$

where $x_{2}^{(U)}$ is the scaling dimension of the four-fermion interaction operator in the disorder-averaged $(2+0)$-D theory.

The dynamic critical exponent $z$ for the disorder-averaged noninteracting model at zero temperature is uniquely determined by the critical behavior of the density of states (DoS). In particular, the DoS scaling dimension $x_{1}=2-z$ [Eq. (2.29)]. Combining this with Eqs. (C3) and (C4) gives Eq. (2.47).

\section{APPENDIX D: CLASS AIII WZNW-FNLSM INTERACTION-STABILIZED NONTRIVIAL FIXED POINT}

The interaction flow equations $(2.65 \mathrm{c})$ and $(2.65 \mathrm{~d})$ possess a nontrivial fixed point. Setting $\lambda=1 / k$ and working to order $\left\{\gamma_{c} / k, \gamma_{c} \lambda_{A}, \gamma_{c}^{2}\right\}$, the fixed point is located at

$$
\begin{aligned}
\gamma_{t}^{*} & =\frac{\lambda_{A}\left(2 k \lambda_{A}-1\right)\left(3 k \lambda_{A}-2\right)}{2\left(k \lambda_{A}-1\right)\left[\lambda_{A}\left(3 k \lambda_{A}+k-2\right)-1\right]}, \\
\gamma_{c}^{*} & =\frac{\lambda_{A}\left(2-3 k \lambda_{A}\right)}{2\left(k \lambda_{A}-1\right)} .
\end{aligned}
$$

[1] For a recent review, see, e.g., J. Alicea, Rep. Prog. Phys. 75, 076501 (2012).

[2] A. P. Schnyder, S. Ryu, A. Furusaki, and A. W. W. Ludwig, Phys. Rev. B 78, 195125 (2008).

[3] A. Kitaev, AIP Conf. Proc. No. 1134 (AIP, New York, 2009), p. 22.

[4] A. P. Schnyder, S. Ryu, and A. W. W. Ludwig, Phys. Rev. Lett. 102, 196804 (2009).

[5] S. Ryu, A. P. Schnyder, A. Furusaki, and A. W. W. Ludwig, New J. Phys. 12, 065010 (2010).

[6] M. Z. Hasan and C. L. Kane, Rev. Mod. Phys. 82, 3045 (2010); X.-L. Qi and S.-C. Zhang, ibid. 83, 1057 (2011).

[7] R. Roy, arXiv:0803.2868.

[8] X.-L. Qi, T. L. Hughes, S. Raghu, and S.-C. Zhang, Phys. Rev. Lett. 102, 187001 (2009).

[9] L. Fu and E. Berg, Phys. Rev. Lett. 105, 097001 (2010).

[10] A. P. Schnyder, P. M. R. Brydon, D. Manske, and C. Timm, Phys. Rev. B 82, 184508 (2010).

[11] K. Yada, M. Sato, Y. Tanaka, and T. Yokoyama, Phys. Rev. B 83, 064505 (2011).

[12] A. Yamakage, K. Yada, M. Sato, and Y. Tanaka, Phys. Rev. B 85, 180509(R) (2012).

[13] Y.-Y. Xiang, W.-S. Wang, Q.-H. Wang, and D.-H. Lee, Phys. Rev. B 86, 024523 (2012).

[14] T. H. Hsieh and L. Fu, Phys. Rev. Lett. 108, 107005 (2012).

[15] M. S. Foster and E. A. Yuzbashyan, Phys. Rev. Lett. 109, 246801 (2012).

[16] G. E. Volovik, The Universe in a Helium Droplet (Oxford University Press, Oxford, 2003).

[17] D. Bernard and A. LeClair, J. Phys. A 35, 2555 (2002).

[18] R. Nakai and K. Nomura, Phys. Rev. B 89, 064503 (2014).

[19] A. W. W. Ludwig, M. P. A. Fisher, R. Shankar, and G. Grinstein, Phys. Rev. B 50, 7526 (1994).

[20] A. A. Nersesyan, A. M. Tsvelik, and F. Wenger, Phys. Rev. Lett. 72, 2628 (1994); Nucl. Phys. 438, 561 (1995).

[21] G. Paladin and A. Vulpiani, Phys. Rep. 156, 147 (1987).

[22] For reviews, see B. Huckestein, Rev. Mod. Phys. 67, 357 (1995); M. Janssen, Int. J. Mod. Phys. B 8, 943 (1994).

[23] F. Evers and A. D. Mirlin, Rev. Mod. Phys. 80, 1355 (2008).

[24] C. C. Chamon, C. Mudry, and X.-G. Wen, Phys. Rev. Lett. 77, 4194 (1996).

[25] H. E. Castillo, C. C. Chamon, E. Fradkin, P. M. Goldbart, and C. Mudry, Phys. Rev. B 56, 10668 (1997).

[26] D. Carpentier and P. Le Doussal, Phys. Rev. E 63, 026110 (2001).

[27] O. Motrunich, K. Damle, and D. A. Huse, Phys. Rev. B 65, 064206 (2002).
[28] B. Horovitz and P. Le Doussal, Phys. Rev. B 65, 125323 (2002).

[29] C. Mudry, S. Ryu, and A. Furusaki, Phys. Rev. B 67, 064202 (2003).

[30] A. M. Tsvelik, Phys. Rev. B 51, 9449 (1995).

[31] D. Bernard, (Perturbed) conformal field theory applied to $2 d$ disordered systems: an introduction, in Low-Dimensional Applications of Quantum Field Theory: Proceedings of a NATO ASI Held in Cargese, France, July 11-29, 1995, edited by L. Baulieu, V. Kazakov, and M. Picco, NATO Science Series: Physics B Vol. 362 (Plenum Press, New York, 1997); arXiv:hepth/9509137.

[32] C. Mudry, C. Chamon, and X.-G. Wen, Nucl. Phys. B 466, 383 (1996).

[33] J. S. Caux, I. I. Kogan, and A. M. Tsvelik, Nucl. Phys. B 466, 444 (1996).

[34] A. W. W. Ludwig, arXiv:cond-mat/0012189.

[35] M. J. Bhaseen, J.-S. Caux, I. I. Kogan, and A. M. Tsvelik, Nucl. Phys. B 618, 465 (2001).

[36] A. LeClair and D. J. Robinson, J. Phys. A 41, 452002 (2008).

[37] J. T. Chalker and G. J. Daniell, Phys. Rev. Lett. 61, 593 (1988).

[38] J. Chalker, Physica A 167, 253 (1990).

[39] E. Cuevas and V. E. Kravtsov, Phys. Rev. B 76, 235119 (2007).

[40] T. I. Baturina et al., Physica C 468, 316 (2008); M. V. Feigel'man, L. B. Ioffe, V. E. Kravtsov, and E. A. Yuzbashyan, Phys. Rev. Lett. 98, 027001 (2007).

[41] M. V. Feigel'man, L. B. Ioffe, V. E. Kravtsov, and E. Cuevas, Ann. Phys. 325, 1390 (2010).

[42] D. Basko, I. Aleiner, and B. Altshuler, Ann. Phys. 321, 1126 (2006).

[43] In Fig. 1, we plot 2D wave functions obtained by exact diagonalization of a class BDI nearest-neighbor random hopping model on the square lattice. The size of the system is $44 \times 44$, with periodic boundary conditions. This is a nontopological system that exhibits critically delocalized states near zero energy, due to an artificial chiral symmetry associated to the special form of the disorder [23]. The probability densities for the first four positive energy states are shown in (a), while four successive localized states near the upper band edge are shown in (b). The surface states of a class CI topological superconductor have the same qualitative features: critical delocalization at zero energy, and localization away from this. (The crucial difference is that the special form of the disorder in the latter case is determined by the topology, rather than by fine-tuning on the lattice scale.) We plot wave functions for the 2D BDI model because they entail less computation than class CI surface states. To obtain 
the latter, one must in principle diagonalize a surface-disordered 3D bulk model [4,44] (but see, e.g., Ref. [61]).

[44] G. Schubert, H. Fehske, L. Fritz, and M. Vojta, Phys. Rev. B 85, 201105(R) (2012).

[45] P. Di Francesco, P. Mathieu, and D. Sénéchal, Conformal Field Theory (Springer-Verlag, New York, 1996).

[46] A. M. Finkel'stein, Zh. Eksp. Teor. Fiz. 84, 168 (1983) [Sov. Phys. JETP 57, 97 (1983)].

[47] For a review, see, e.g., D. Belitz and T. R. Kirkpatrick, Rev. Mod. Phys. 66, 261 (1994).

[48] M. S. Foster and A. W. W. Ludwig, Phys. Rev. B 74, 241102(R) (2006).

[49] L. Dell'Anna, Nucl. Phys. B 758, 255 (2006).

[50] M. S. Foster and A. W. W. Ludwig, Phys. Rev. B 77, 165108 (2008).

[51] H.-Y. Xie, Y.-Z. Chou, and M. S. Foster (unpublished).

[52] L. Fidkowski, X. Chen, and A. Vishwanath, Phys. Rev. X 3, 041016 (2013).

[53] C. Wang, A. C. Potter, and T. Senthil, Science 343, 629 (2014).

[54] C. Wang and T. Senthil, arXiv:1401.1142.

[55] P. M. Ostrovsky, I. V. Gornyi, and A. D. Mirlin, Phys. Rev. Lett. 105, 036803 (2010).

[56] R. Nandkishore, J. Maciejko, D. A. Huse, and S. L. Sondhi, Phys. Rev. B 87, 174511 (2013).

[57] Y. Frishman and J. Sonnenschein, Phys. Rep. 223, 309 (1993).

[58] J. Fuchs, Affine Lie Algebras and Quantum Groups (Cambridge University Press, Cambridge, England, 1992).

[59] A. Altland and B. Simons, Condensed Matter Field Theory (Cambridge University Press, Cambridge, England, 2010).

[60] K. Efetov, Supersymmetry in Disorder and Chaos (Cambridge University Press, Cambridge, England, 1999).

[61] Y.-Z. Chou and M. S. Foster, Phys. Rev. B (to be published), arXiv:1402.6797.

[62] I. Affleck, in Fields, Strings and Critical Phenomena, edited by E. Brezin and J. Zinn-Justin, Proceedings of the Les Houches Summer School, Session XLIX (North-Holland, Amsterdam, 1988).

[63] A. Altland, B. D. Simons, and M. R. Zirnbauer, Phys. Rep. 359, 283 (2002)

[64] P. M. Ostrovsky, I. V. Gornyi, and A. D. Mirlin, Phys. Rev. B 74, 235443 (2006).

[65] Y. Hatsugai, X.-G. Wen, and M. Kohmoto, Phys. Rev. B 56, 1061 (1997); Y. Morita and Y. Hatsugai, Phys. Rev. Lett. 79, 3728 (1997).

[66] S. Ryu and Y. Hatsugai, Phys. Rev. B 63, 233307 (2001); 65, 033301 (2001).

[67] L. A. Wray, S.-Y. Xu, Y. Xia, Y. S. Hor, D. Qian, A. V. Fedorov, H. Lin, A. Bansil, R. J. Cava, and M. Z. Hasan, Nat. Phys. 6, 855 (2010)

[68] S. Sasaki, M. Kriener, K. Segawa, K. Yada, Y. Tanaka, M. Sato, and Y. Ando, Phys. Rev. Lett. 107, 217001 (2011).

[69] T. V. Bay, T. Naka, Y. K. Huang, H. Luigjes, M. S. Golden, and A. de Visser, Phys. Rev. Lett. 108, 057001 (2012).

[70] X. Chen, C. Huan, Y. S. Hor, C. A. R. Sá de Melo, and Z. Jiang, arXiv:1210.6054.

[71] H. Peng, D. De, B. Lv, F. Wei, and C.-W. Chu, Phys. Rev. B 88, 024515 (2013).

[72] S. Ryu, J. E. Moore, and A. W. W. Ludwig, Phys. Rev. B 85, 045104 (2012).
[73] M. Stone, Phys. Rev. B 85, 184503 (2012).

[74] I. A. Gruzberg, A. W. W. Ludwig, and N. Read, Phys. Rev. Lett. 82, 4524 (1999); T. Senthil, J. B. Marston, and M. P. A. Fisher, Phys. Rev. B 60, 4245 (1999).

[75] N. Read and D. Green, Phys. Rev. B 61, 10267 (2000).

[76] D.-H. Lee and Z. Wang, Phys. Rev. Lett. 76, 4014 (1996).

[77] J. Cardy, Scaling and Renormalization in Statistical Physics (Cambridge University Press, Cambridge, England, 1996).

[78] See, e.g., A. A. Fedorenko, D. Carpentier, and E. Orignac, Phys. Rev. B 85, 125437 (2012) and references therein.

[79] In a $(2+1)$-D formulation of the theory, the density of states operator in imaginary time is local in space and Matsubara frequencies: $v(\varepsilon, \mathbf{r}) \sim \psi^{\dagger}\left(\varepsilon_{n}, \mathbf{r}\right) \psi\left(\varepsilon_{n}, \mathbf{r}\right)$.

[80] T. Senthil and M. P. A. Fisher, Phys. Rev. B 61, 9690 (2000).

[81] M. S. Foster, S. Ryu, and A. W. W. Ludwig, Phys. Rev. B 80, 075101 (2009).

[82] A. D. Mirlin, Y. V. Fyodorov, A. Mildenberger, and F. Evers, Phys. Rev. Lett. 97, 046803 (2006).

[83] I. A. Gruzberg, A. W. W. Ludwig, A. D. Mirlin, and M. R. Zirnbauer, Phys. Rev. Lett. 107, 086403 (2011).

[84] I. A. Gruzberg, A. D. Mirlin, and M. R. Zirnbauer, Phys. Rev. B 87, 125144 (2013).

[85] From Ref. [15], for class CI one has

$$
x_{q}=\frac{(2-q) q}{2(k+1)},
$$

which satisfies Eq. (2.37) with $q_{*}=2$. From Eq. (2.73) in the present paper, the result for DIII is

$$
x_{q}=-\frac{q^{2}}{(k-2)},
$$

which has $q_{*}=0$. These are consistent with Refs. [83,84].

[86] A. Richardella et al., Science 327, 665 (2010).

[87] I. S. Burmistrov, I. V. Gornyi, and A. D. Mirlin, Phys. Rev. Lett. 111, 066601 (2013).

[88] M. S. Foster, Phys. Rev. B 85, 085122 (2012).

[89] E. J. König, P. M. Ostrovsky, I. V. Protopopov, and A. D. Mirlin, Phys. Rev. B 85, 195130 (2012).

[90] The theory with $\lambda \neq 1 / k$ should be referred to as a principal chiral model plus a Wess-Zumino-Novikov-Witten term.

[91] E. Witten, Comm. Math. Phys. 92, 455 (1984).

[92] S. Guruswamy, A. LeClair, and A. W. W. Ludwig, Nucl. Phys. B 583, 475 (2000).

[93] R. Gade and F. Wegner, Nucl. Phys. B 360, 213 (1991); R. Gade, ibid. 398, 499 (1993).

[94] B. Duplantier and A. W. W. Ludwig, Phys. Rev. Lett. 66, 247 (1991).

[95] T. Senthil, M. P. A. Fisher, L. Balents, and C. Nayak, Phys. Rev. Lett. 81, 4704 (1998).

[96] B. L. Altshuler and A. G. Aronov, in Electron-Electron Interactions in Disordered Systems, edited by A. L. Efros and M. Pollak (Elsevier, North Holland, 1985), p. 1.

[97] In principle, one can obtain results to higher orders in the Cooper channel interaction $\gamma_{c}$ in a one-loop Finkel'stein sigma model calculation. However, substantial technical difficulties arise [47], and for this reason one typically works only to the lowest nontrivial order.

[98] P. W. Anderson, J. Phys. Chem. Solids 11, 26 (1959).

[99] M. S. Foster and I. L. Aleiner, Phys. Rev. B 77, 195413 (2008). 